\title{
A Hierarchy of Reverse Bisimulations on Stable Configuration Structures
}

\author{
IAIN PHILLIPS ${ }^{1}$ and IREK ULIDOWKI \\ ${ }^{1}$ Department of Computing, Imperial College London, \\ 180 Queen's Gate, London SW7 2AZ, England \\ Email: iccp@doc.ic.ac.uk \\ ${ }^{2}$ Department of Computer Science, University of Leicester, \\ University Road, Leicester LE1 7RH, England \\ Email: iu3@mcs.le.ac.uk
}

Received 7 April 2010

\begin{abstract}
The relationships between various equivalences on stable configuration structures, including interleaving bisimulation (IB), step bisimulation (SB), pomset bisimulation and hereditary history-preserving (H-H) bisimulation, have been investigated by van Glabbeek and Goltz (and later Fecher). Since H-H bisimulation may be characterised by the use of reverse as well as forward transitions, it is of interest to investigate these and other forms of bisimulations where both forward and reverse transitions are allowed. Bednarczyk asked whether SB with reverse steps is as strong as H-H bisimulation. We answer this question negatively. We give various characterisations of SB with reverse steps, showing that forward steps do not add power. We strengthen Bednarczyk's result that, in the absence of auto-concurrency, reverse IB is as strong as $\mathrm{H}-\mathrm{H}$ bisimulation, by showing that we need only exclude auto-concurrent events at the same depth in the configuration.

We consider several other forms of observations of reversible behaviour. We define a wide range of bisimulations by mixing the forward and reverse observations. We investigate the power of these bisimulations and represent the relationships between them as a hierarchy which has IB at the bottom and $\mathrm{H}-\mathrm{H}$ at the top.
\end{abstract}

\section{Introduction}

The relationships between various equivalences on configuration structures, including interleaving bisimulation (IB), step bisimulation (SB), pomset bisimulation (PB) and hereditary history-preserving $(\mathrm{H}-\mathrm{H})$ bisimulation, have been investigated by van Glabbeek and Goltz (2001) (and later Fecher (2004)). Since H-H bisimulation may be characterised by the use of reverse as well as forward transitions, it is of interest to investigate forms of IB, SB and PB where both forward and reverse transitions are allowed. We shall look at the various possible combinations, such as forward pomsets with reverse steps, and map out the hierarchy of equivalences induced by such notions.

In this paper we adopt as our model of concurrency the framework of stable configuration structures, following van Glabbeek and Goltz. These can be regarded as a 
more abstract version of stable event structures (Winskel 1987). A configuration is a set of events which is a possible run within an event structure. Using stable configuration structures means that our results can be directly related to those of van Glabbeek and Goltz, and of Fecher. In our earlier work on reversible computation (Phillips and Ulidowski 2007a) we employed prime event structures, which are a special case of stable ESs. Prime event structures are technically simpler; they have a global causal ordering on events, whereas with stable event structures causal orderings are parametrised by configuration. Stable event structures have the advantage over prime event structures that such operations as sequential and parallel composition can be modelled more easily. See e.g. (van Glabbeek and Goltz 2001) for further discussion of the merits of the various forms of event structure.

The question then arises as to when two configuration structures should be regarded as equivalent. We do not want to distinguish configuration structures merely on the basis that they have different sets of events. So we assume that events come with a labelling (we use $a, b, c$ for labels); events with the same label are equivalent. The idea is that the labels represent what we can observe of the events. Simple configuration structures can be written in a CCS-style notation (Milner 1989), with conflict represented by choice $(+)$, concurrency by parallel composition $(\mid)$, and causal ordering by action prefixing (.). As a simple example, the law $a+a=a$ will hold for any reasonable equivalence on configuration structures, even though the configuration structure represented by $a+a$ has two (conflicting) events while $a$ only has one.

Hereditary history-preserving $(\mathrm{H}-\mathrm{H})$ bisimulation is considered as the finest well-behaved true concurrency equivalence relation that "completely respects the causal and branching structure of concurrent systems and their interplay" (van Glabbeek and Goltz 2001). Here "well-behaved" means that it preserves refinement of actions. H-H bisimulation was proposed by Bednarczyk (1991). It can be regarded as the canonical equivalence on event structures, in view of its category-theoretical characterisation as open map bisimulation with labelled partial orders as the observations (Joyal et al. 1996). H-H bisimulation is defined over configuration structures and, in addition to matching configurations and the transitions between configurations, it also keeps a history of the matched events along matching computations. This is achieved by means of label-preserving and orderpreserving isomorphisms between the elapsed events of the two configuration structures. H-H bisimulation and its decidability were further researched by Fröschle (2004) and in her subsequent papers, for example (Fröschle and Lasota 2005), and by Jurdzinski, Nielsen and Srba (2003).

The work presented in this paper is all motivated by the issue of what observations we can make of concurrent processes. The most fundamental observation is of the label of a single event. This yields interleaving bisimulation (IB), which, as its name indicates, is unable to make true concurrency distinctions, such as between $a \mid b$ and $a . b+b . a$. A popular method for increasing the discriminating power of a bisimulation is to generalise single action transitions $X \stackrel{a}{\rightarrow} X^{\prime}$ to transitions $X \stackrel{\mu}{\rightarrow} X^{\prime}$ where $\mu$ is a structure richer than a single action (Pomello 1986; Boudol and Castellani 1987; Cherief 1992). It could be a set of events occurring concurrently (or a multiset of action labels of these events), a so-called 
"step", giving step bisimulation (SB), or even a pomset, giving pomset bisimulation (PB). Here steps and pomsets are more refined observations that we can make of a process.

As just stated, $\mathrm{H}-\mathrm{H}$ can be regarded as the canonical true concurrency equivalence. However we do not regard this as being observational in character, due to its reliance on order isomorphisms. So then the question arises (and this is the main question underlying the work in this paper) as to whether there is an observational characterisation of $\mathrm{H}-\mathrm{H}$. This is somewhat reminiscent of the programme carried out by Abramsky to see whether observational equivalence (for, say, CCS) can be characterised as a testing equivalence, if the tests are sufficiently powerful (Abramsky 1987).

$\mathrm{H}-\mathrm{H}$ bisimulation requires the isomorphisms to be consistent under both forward and backward transitions between configurations. It is therefore natural to look at the power of forms of IB, SB, and PB where reverse transitions are allowed as well as forward ones. Adding reverse transitions to IB gives us what we shall call reverse interleaving$I B$ (RI-IB). Adding reverse step transitions to $\mathrm{SB}$ gives us reverse step SB (RS-SB). Adding reverse pomset transitions to $\mathrm{PB}$ gives us reverse pomset $P B$ (RP-PB). These three equivalences are the main ones studied in this paper, reflecting the possibility of making observations in both forward and reverse directions. We wish to investigate what extra power this gives.

RI-IB was already investigated by Bednarczyk (1991). When there is no auto-concurrency (concurrent events with the same label), RI-IB equivalence is finer than many true concurrency bisimulations (van Glabbeek and Goltz 2001) up to and including historypreserving bisimulation (De Nicola et al. 1987; Rabinovich and Trakhtenbrot 1988). The so-called absorption law (Boudol and Castellani 1987; Bednarczyk 1991; van Glabbeek and Goltz 2001)

$$
(a \mid(b+c))+(a \mid b)+((a+c) \mid b)=(a \mid(b+c))+((a+c) \mid b)
$$

is not valid for RI-IB equivalence: If one performs $a$ and then $b$ with the $a \mid b$ component on the left, then these must be matched by the $a$ and then the $b$ of the $((a+c) \mid b)$ summand on the right. (Matching it with the $a$ of $(a \mid(b+c))$ is wrong, as after this $a$ is performed, no $c$ is possible after $a$ in $a \mid b$.) The right hand side can now reverse $a$ and do a $c$ (still using the same summand as all other summands are disabled). The left hand side cannot match this.

In fact, Bednarczyk proved that, in the absence of auto-concurrency, RI-IB equivalence has the same power as $\mathrm{H}-\mathrm{H}$ equivalence (on prime event structures). We shall prove (Theorem 9.7) an extension of this result: RI-IB equivalence has the same power as $\mathrm{H}-\mathrm{H}$ equivalence in the absence of equidepth auto-concurrency, i.e. when we cannot have two events with the same label occurring at the same depth within a configuration. The depth of an event $e$ is the length of the longest causal chain of events up to and including $e$.

When auto-concurrency is present, RI-IB is unable to distinguish such simple processes as $a \mid a$ and $a . a$. This motivates study of RS-SB. RS-SB was briefly mentioned by Bednarczyk; he asked the following question: is RS-SB as fine as H-H bisimulation? We answer this question in the negative. In fact we can go further, and show that RP-PB is strictly finer than RS-SB, and $\mathrm{H}-\mathrm{H}$ is strictly finer than RP-PB. This of course means that adding reverse observations (or at least those considered here) is not sufficient to 
give an observational characterisation of H-H. Nevertheless, it is clear that reverse observations add considerable power, as shown by the Absorption Law example above. See Figure 25 in the last section for a diagram showing how RI-IB, RS-SB and RP-PB relate to the hierarchy of forward-only equivalences established by van Glabbeek and Goltz and by Fecher.

If we allow forward step transitions, but only single reverse transitions, then we already can distinguish $a \mid a$ from a.a very easily: $a \mid a$ can do an $\{a, a\}$ step whereas a.a cannot. However we cannot distinguish $a \mid a$ from $(a \mid a)+a . a$. Here the reverse steps are needed, and $a \mid a$ is not equivalent to $(a \mid a)+a . a$ for RS-IB; in $(a \mid a)+a . a$ we can perform two as in sequence and get to a configuration where we cannot do a reverse step $\{a, a\}$, unlike for $a \mid a$.

We show that all the power of RS-SB equivalence resides in the reverse step transitions, with the forward steps being dispensable (though of course often useful in examples). In fact, the reverse steps can be restricted to those which are homogeneous, by which we mean that all events have the same label (thus the events are auto-concurrent) (Theorem 5.10). One can even restrict attention to reverse homogeneous equidepth steps, where all events have the same depth. We also show that RS-SB equivalence preserves depth, in the sense that corresponding events must have the same depth.

We introduce the notion of depth-respecting bisimulation (DB). This is inspired by the idea that instead of observing steps or pomsets, one can give oneself the power to observe the depth of events. In the forward-only direction, it turns out that DB is strictly stronger than SB. However we prove that reverse depth-respecting DB (RD-DB) has the same power as RS-SB (Theorem 5.24). We also use DB to give a simpler proof of Fecher's result that weak history-preserving equivalence is included in SB equivalence. We concede that the depth of an event is a less plausible observation than a step or a pomset, and to some extent DB and its variants are a technical matter. Nevertheless, it is interesting that observing depth is precisely equivalent to observing steps, when both forward and reverse observations are allowed.

One of the main purposes van Glabbeek and Goltz had in studying bisimulation-based equivalences on stable configuration structures was to identify equivalences which are preserved by refinement of actions. Of the equivalences we discuss, they identified two which are preserved by refinement, namely history-preserving bisimulation and hereditary history-preserving bisimulation. We round out their study by showing (Proposition 8.1) that all the reverse-type equivalences considered here fail to be preserved by refinement, with the possible exception of those few ones which are sandwiched between historypreserving bisimulation and hereditary history-preserving bisimulation; see Figure 24 in the last section. Thus Proposition 8.1 shows that there is a trade-off between the observational character of an equivalence and its preservation by refinement of actions.

The paper is organised as follows. We start by defining stable configuration structures in Section 2. Then in Section 3 we look at forward-only equivalences, including new depthrespecting equivalences. Section 4 is devoted to equivalences enhanced with the power of reversing single events. In Section 5 we look at equivalences with reverse steps, and show among other results that these are equivalent to reverse depth-respecting equivalences, and that they give the power of forward steps. We briefly investigate equivalences with 
reverse pomsets in Section 6, and those with the hereditary property in Section 7. Section 8 briefly discusses which equivalences are preserved under refinement. In Section 9 we show that Bednarczyk's result described above still holds in the absence of equidepth auto-concurrency, and we look at how the hierarchy of equivalences collapses under this assumption. We then draw some conclusions.

\section{Configuration structures and prime event structures}

In this section we define our computational model, namely configuration structures. We start by defining prime event structures, since this is a better-known formalism, and we shall phrase most of our examples as prime event structures.

\subsection{Prime event structures}

We assume a set of action labels Act, ranged over by $a, b, \ldots$

Definition 2.1. (Nielsen et al. 1981) A (labelled) prime event structure is a 4-tuple $\mathcal{E}=(E,<, \sharp, \ell)$ where

$-E$ is a set of events;

$-<\subseteq E \times E$ is an irreflexive partial order (the causality relation) such that for any $e \in E$, the set $\left\{e^{\prime} \in E: e^{\prime}<e\right\}$ is finite;

$-\sharp \subseteq E \times E$ is an irreflexive, symmetric relation (the conflict relation) such that if $e_{1}<e_{2}$ and $e_{1} \sharp e$ then $e_{2} \sharp e$;

$-\ell: E \rightarrow$ Act is the labelling function.

As we have defined it, prime event structures have binary conflict, though this can be generalised to non-binary conflict, where, say, out of three events only two can occur (van Glabbeek and Vaandrager 1997).

When drawing diagrams of event structures we shall, as usual, depict the causal relation with arrows, and the conflict relation with dotted lines. We shall also suppress the actual events and write their labels instead. Thus if we have two events $e_{1}$ and $e_{2}$, both labelled with $a$, in diagrams we shall denote them both as $a$, or sometimes as $a_{1}$ and $a_{2}$, respectively, when we wish to distinguish between them. This is justified, since all the notions of equivalence we shall discuss depend on the labels of the events, rather than the events themselves.

Definition 2.2. Let $\mathcal{E}=(E,<, \sharp, \ell)$ and let $X \subseteq E$.

$-X$ is conflict-free if $\sharp \cap(X \times X)=\emptyset$;

$-X$ is left-closed if whenever $e \in X$ and $e^{\prime}<e$ then $e^{\prime} \in X$;

$-X$ is a configuration of $\mathcal{E}$ if $X$ is finite, left-closed and conflict-free.

\subsection{Configuration structures}

We define configuration structures much as in (van Glabbeek and Goltz 2001), with the omission of the termination predicate for simplicity. We keep as close as possible to (van Glabbeek and Goltz 2001) in most of the definitions in this section. 


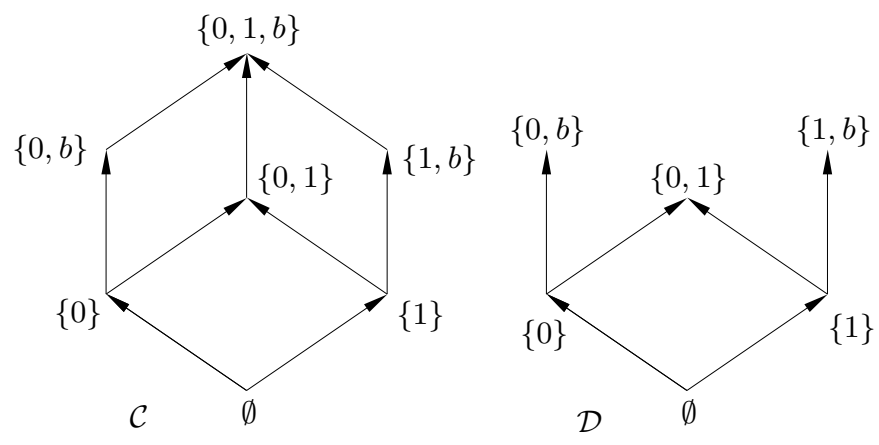

Fig. 1. Non-stable and stable configuration structures.

Definition 2.3. A configuration structure (over an alphabet Act) is a pair $\mathcal{C}=(C, \ell)$ where $C$ is a family of finite sets (configurations) and $\ell: \bigcup_{X \in C} X \rightarrow$ Act is a labelling function.

We use $C_{\mathcal{C}}, \ell_{\mathcal{C}}$ to refer to the two components of a configuration structure $\mathcal{C}$. Also we let $E_{\mathcal{C}}=\bigcup_{X \in C} X$, the events of $\mathcal{C}$. We let $e, \ldots$ range over events, and $E, F, \ldots$ over sets of events.

Definition 2.4. (van Glabbeek and Goltz 2001) A configuration structure $\mathcal{C}=(C, \ell)$ is stable if it is

- rooted: $\emptyset \in C$;

— connected: $\emptyset \neq X \in C$ implies $\exists e \in X: X \backslash\{e\} \in C$;

- closed under bounded unions: if $X, Y, Z \in C$ then $X \cup Y \subseteq Z$ implies $X \cup Y \in C$;

- closed under bounded intersections: if $X, Y, Z \in C$ then $X \cup Y \subseteq Z$ implies $X \cap Y \in C$.

Note that in the presence of the "closed under bounded unions" condition the "closed under bounded intersections" condition can be simplified to "if $X, Y, X \cup Y \in C$ then $X \cap Y \in C^{\prime \prime}$.

Any stable configuration structure is the set of configurations of a stable event structure (van Glabbeek and Goltz 2001, Theorem 5.3).$^{\dagger}$ In Figure 1 we give two example configuration structures derived from examples of Winskel (1987). In each of $\mathcal{C}, \mathcal{D}$ the labelling can be taken to be the identity function. Configuration structure $\mathcal{C}$ models a "parallel switch" where events 0 or 1 can light the bulb $b$. If both 0 and 1 occur then $b$ can also happen. This is inclusive "or" causation. We see that $\mathcal{C}$ is not stable, since it is not closed under bounded intersections: $\{0, b\} \cup\{1, b\}$ is bounded by $\{0,1, b\}$, but $\{0, b\} \cap\{1, b\}=\{b\} \notin C_{\mathcal{C}}$. By contrast, $\mathcal{D}$ is stable; it models a switch where the bulb can be lit by either 0 or 1 , but not both, i.e. exclusive "or" causation.

Configuration structures have associated notions of causal orderings on events and concurrency between events:

\footnotetext{
$\dagger$ We work with stable configuration structures alone and never directly with stable event structures. The definition of stable event structures can be found in (Winskel 1987; van Glabbeek and Goltz 2001).
} 
Definition 2.5. Let $\mathcal{C}=(C, \ell)$ be a stable configuration structure, and let $X \in C$.

- Causality: $d \leq_{X} e$ iff for all $Y \in C$ with $Y \subseteq X$ we have $e \in Y$ implies $d \in Y$. Furthermore $d<_{X} e$ iff $d \leq_{X} e$ and $d \neq e$.

- Concurrency: $d \operatorname{co}_{X} e$ iff $d \nless_{X} e$ and $e \nless x d$.

It is shown in (van Glabbeek and Goltz 2001) that $<_{X}$ is a partial order and that the sub-configurations of $X$ are precisely those subsets $Y$ which are left-closed w.r.t. $<_{X}$, i.e. if $d<_{X} e \in Y$ then $d \in Y$. Furthermore, if $X, Y \in C$ with $Y \subseteq X$, then $<_{Y}=<_{X}\lceil Y$.

The set of configurations of a prime event structure forms a configuration structure, as is easy to check. In fact prime event structures form a proper subclass of stable event structures; the configuration structures associated with prime event structures are got by strengthening the "closed under bounded intersections" condition of Definition 2.3 to closure under intersections: if $X, Y \in C$ then $X \cap Y \in C$ (van Glabbeek 1996). In Figure 1, $\mathcal{D}$ is not prime, since it is not closed under intersections: $\{0, b\} \cap\{1, b\}=\{b\} \notin C_{\mathcal{D}}$. Thus prime event structures do not allow "or" causation; to model the switch as a prime event structure we would have to model the lighting of the bulb as two separate events, one caused by 0 and the other by 1 .

\section{Forward-only equivalences}

We investigate the hierarchy of forward-only bisimulation-based equivalences. We start by recalling existing equivalences (Section 3.1), and then we introduce new depth-respecting and homogeneous equivalences in Sections 3.2 and 3.3. Depth-respecting bisimulation, while of lesser interest in its own right, will help us obtain a simpler proof of Fecher's result that weak history-preserving equivalence is included in SB equivalence. So-called homogeneous variants of step bisimulation are again of lesser interest in their own right, but are needed in later technical development in Sections 5 and 9.

\subsection{Existing equivalences}

We now recall the definition of various notions of equivalence between configuration structures. We start by defining the most basic labelled transition relation, on single events.

Definition 3.1. Let $\mathcal{C}=(C, \ell)$ be a stable configuration structure and let $a \in$ Act. We let $X \stackrel{a}{\rightarrow} \mathcal{C} X^{\prime}$ iff $X, X^{\prime} \in C, X \subseteq X^{\prime}$ and $X^{\prime} \backslash X=\{e\}$ with $\ell(e)=a$.

We shall depict many of our example configuration structures as labelled transition diagrams where the nodes are configurations, with the empty configuration as the start node. We tend not to give the actual configurations as sets of events; clearly these can be recovered from such diagrams, up to isomorphism.

Definition 3.2. (van Glabbeek and Goltz 2001) Let $\mathcal{C}, \mathcal{D}$ be stable configuration structures. A relation $R \subseteq C_{\mathcal{C}} \times C_{\mathcal{D}}$ is an interleaving bisimulation (IB) between $\mathcal{C}$ and $\mathcal{D}$ if $(\emptyset, \emptyset) \in R$ and if $(X, Y) \in R$ then for $a \in$ Act 
— if $X \stackrel{a}{\rightarrow} \mathcal{C} X^{\prime}$ then $\exists Y^{\prime} . Y \stackrel{a}{\rightarrow} \mathcal{D} Y^{\prime}$ and $\left(X^{\prime}, Y^{\prime}\right) \in R$;

- if $Y \stackrel{a}{\rightarrow} \mathcal{D} Y^{\prime}$ then $\exists X^{\prime} . X \stackrel{a}{\rightarrow} \mathcal{C} X^{\prime}$ and $\left(X^{\prime}, Y^{\prime}\right) \in R$.

We say that $\mathcal{C}$ and $\mathcal{D}$ are IB equivalent $\left(\mathcal{C} \approx_{i b} \mathcal{D}\right)$ iff there is an IB between $\mathcal{C}$ and $\mathcal{D}$.

For a set of events $E$, let $\ell(E)$ be the multiset of labels of events in $E$. We define a step transition relation where concurrent events are executed in a single step:

Definition 3.3. Let $\mathcal{C}=(C, \ell)$ be a stable configuration structure and assume $A \in \mathbb{N}^{\text {Act }}$ ( $A$ is a multiset over Act). We let $X \stackrel{A}{\rightarrow} \mathcal{C} X^{\prime}$ iff $X, X^{\prime} \in C, X \subseteq X^{\prime}$, and $X^{\prime} \backslash X=E$ with $d c_{X^{\prime}} e$ for all $d, e \in E$ and $\ell(E)=A$.

Definition 3.4. (Pomello 1986; van Glabbeek and Goltz 2001) Let $\mathcal{C}, \mathcal{D}$ be stable configuration structures. A relation $R \subseteq C_{\mathcal{C}} \times C_{\mathcal{D}}$ is a step bisimulation (SB) between $\mathcal{C}$ and $\mathcal{D}$ if $(\emptyset, \emptyset) \in R$ and if $(X, Y) \in R$ then for $A \in \mathbb{N}^{\text {Act }}$

- if $X \stackrel{A}{\rightarrow} \mathcal{C} X^{\prime}$ then $\exists Y^{\prime} . Y \stackrel{A}{\rightarrow}_{\mathcal{D}} Y^{\prime}$ and $\left(X^{\prime}, Y^{\prime}\right) \in R$;

- if $Y \stackrel{A}{\rightarrow} \mathcal{D} Y^{\prime}$ then $\exists X^{\prime} . X \stackrel{A}{\rightarrow} \mathcal{C} X^{\prime}$ and $\left(X^{\prime}, Y^{\prime}\right) \in R$.

We say that $\mathcal{C}$ and $\mathcal{D}$ are $\mathrm{SB}$ equivalent $\left(\mathcal{C} \approx_{s b} \mathcal{D}\right)$ iff there is an $\mathrm{SB}$ between $\mathcal{C}$ and $\mathcal{D}$.

Definition 3.5. Let $\mathcal{X}=\left(X,<_{X}, \ell_{X}\right)$ and $\mathcal{Y}=\left(Y,<_{Y}, \ell_{Y}\right)$ be partial orders which are labelled over Act. We say that $\mathcal{X}$ and $\mathcal{Y}$ are isomorphic $(X \cong Y)$ iff there is a bijection from $X$ to $Y$ respecting the ordering and the labelling. The isomorphism class $[\mathcal{X}] \cong$ of a partial order labelled over Act is called a pomset over Act. We let $u, \ldots$ range over pomsets.

Definition 3.6. Let $\mathcal{C}=(C, \ell)$ be a stable configuration structure and let $u$ be a pomset over Act. We let $X \stackrel{u}{\rightarrow} \mathcal{C} X^{\prime}$ iff $X, X^{\prime} \in C, X \subseteq X^{\prime}$, and $X^{\prime} \backslash X=H$ with

$$
u=\left[\left(H,<_{X^{\prime}} \cap(H \times H), \ell_{\mathcal{C}}\lceil H)\right] \cong .\right.
$$

Definition 3.7. (Boudol and Castellani 1987; van Glabbeek and Goltz 2001) Let $\mathcal{C}, \mathcal{D}$ be stable configuration structures. A relation $R \subseteq C_{\mathcal{C}} \times C_{\mathcal{D}}$ is a pomset bisimulation (PB) between $\mathcal{C}$ and $\mathcal{D}$ if $(\emptyset, \emptyset) \in R$ and if $(X, Y) \in R$ then for $u$ any pomset over Act

- if $X \stackrel{u}{\rightarrow} \mathcal{C} X^{\prime}$ then $\exists Y^{\prime} . Y \stackrel{u}{\rightarrow}_{\mathcal{D}} Y^{\prime}$ and $\left(X^{\prime}, Y^{\prime}\right) \in R$;

- if $Y \stackrel{u}{\rightarrow}_{\mathcal{D}} Y^{\prime}$ then $\exists X^{\prime} . X \stackrel{u}{\rightarrow} \mathcal{C} X^{\prime}$ and $\left(X^{\prime}, Y^{\prime}\right) \in R$.

We say that $\mathcal{C}$ and $\mathcal{D}$ are $\mathrm{PB}$ equivalent $\left(\mathcal{C} \approx_{p b} \mathcal{D}\right)$ iff there is a PB between $\mathcal{C}$ and $\mathcal{D}$.

Definition 3.8. (De Nicola et al. 1987; van Glabbeek and Goltz 2001) Let $\mathcal{C}, \mathcal{D}$ be stable configuration structures. A relation $R \subseteq C_{\mathcal{C}} \times C_{\mathcal{D}}$ is a weak history-preserving $(W H)$ bisimulation between $\mathcal{C}$ and $\mathcal{D}$ if $(\emptyset, \emptyset) \in R$ and if $(X, Y) \in R$ and $a \in$ Act

$-\left(X,<_{X}, \ell_{\mathcal{C}}\lceil X) \cong\left(Y,<_{Y}, \ell_{\mathcal{D}}\lceil Y)\right.\right.$;

- if $X \stackrel{a}{\rightarrow} \mathcal{C} X^{\prime}$ then $\exists Y^{\prime} . Y \stackrel{a}{\rightarrow} \mathcal{D} Y^{\prime}$ and $\left(X^{\prime}, Y^{\prime}\right) \in R$;

- if $Y \stackrel{a}{\rightarrow} \mathcal{D} Y^{\prime}$ then $\exists X^{\prime} . X \stackrel{a}{\rightarrow} \mathcal{C} X^{\prime}$ and $\left(X^{\prime}, Y^{\prime}\right) \in R$.

We say that $\mathcal{C}$ and $\mathcal{D}$ are WH equivalent $\left(\mathcal{C} \approx_{w h} \mathcal{D}\right)$ iff there is a WH bisimulation between $\mathcal{C}$ and $\mathcal{D}$.

We can define a further equivalence by combining pomset and weak-history preserving bisimulation: 
Definition 3.9. (van Glabbeek and Goltz 2001) Let $\mathcal{C}, \mathcal{D}$ be stable configuration structures. A relation $R \subseteq C_{\mathcal{C}} \times C_{\mathcal{D}}$ is a weak history-preserving pomset bisimulation (WHPB) between $\mathcal{C}$ and $\mathcal{D}$ if $(\emptyset, \emptyset) \in R$ and if $(X, Y) \in R$ and $u$ is a pomset over Act then

$-\left(X,<_{X}, \ell_{\mathcal{C}} \mid X\right) \cong\left(Y,<_{Y}, \ell_{\mathcal{D}} \mid Y\right)$;

- if $X \stackrel{u^{\rightarrow}}{\rightarrow} X^{\prime}$ then $\exists Y^{\prime} . Y \stackrel{u}{\rightarrow}_{\mathcal{D}} Y^{\prime}$ and $\left(X^{\prime}, Y^{\prime}\right) \in R$;

- if $Y \stackrel{u}{\rightarrow}_{\mathcal{D}} Y^{\prime}$ then $\exists X^{\prime} . X \stackrel{u}{\rightarrow}_{\mathcal{C}} X^{\prime}$ and $\left(X^{\prime}, Y^{\prime}\right) \in R$.

We say that $\mathcal{C}$ and $\mathcal{D}$ are WHPB equivalent $\left(\mathcal{C} \approx_{w h p b} \mathcal{D}\right)$ iff there is a WHPB between $\mathcal{C}$ and $\mathcal{D}$.

Definition 3.10. (Rabinovich and Trakhtenbrot 1988; van Glabbeek and Goltz 2001) Let $\mathcal{C}, \mathcal{D}$ be stable configuration structures. A relation $R \subseteq C_{\mathcal{C}} \times C_{\mathcal{D}} \times \mathcal{P}\left(E_{\mathcal{C}} \times E_{\mathcal{D}}\right)$ is a history-preserving (H) bisimulation between $\mathcal{C}$ and $\mathcal{D}$ iff $(\emptyset, \emptyset, \emptyset) \in R$ and if $(X, Y, f) \in R$ and $a \in$ Act

- $f$ is an isomorphism between $\left(X,<_{X}, \ell_{\mathcal{C}}\lceil X)\right.$ and $\left(Y,<_{Y}, \ell_{\mathcal{D}}\lceil Y)\right.$;

— if $X \stackrel{a}{\rightarrow} \mathcal{C} X^{\prime}$ then $\exists Y^{\prime}, f^{\prime} . Y \stackrel{a}{\rightarrow} \mathcal{D} Y^{\prime},\left(X^{\prime}, Y^{\prime}, f^{\prime}\right) \in R$ and $f^{\prime} \uparrow X=f$;

- if $Y \stackrel{a}{\rightarrow} \mathcal{D} Y^{\prime}$ then $\exists X^{\prime}, f^{\prime} . X \stackrel{a}{\rightarrow} \mathcal{C} X^{\prime},\left(X^{\prime}, Y^{\prime}, f^{\prime}\right) \in R$ and $f^{\prime} \uparrow X=f$.

We say that $\mathcal{C}$ and $\mathcal{D}$ are $\mathrm{H}$ equivalent $\left(\mathcal{C} \approx_{h} \mathcal{D}\right)$ iff there is an $\mathrm{H}$ bisimulation between $\mathcal{C}$ and $\mathcal{D}$.

It is shown in (van Glabbeek and Goltz 2001) that considering more complex transitions such as step transitions or pomset transitions in the definition of $\mathrm{H}$ bisimulation does not change the resulting equivalence notion.

Proposition 3.11. (van Glabbeek and Goltz 2001) On stable configuration structures, $\approx_{h} \subsetneq \approx_{w h p b} \subsetneq \approx_{p b} \subsetneq \approx_{s b} \subsetneq \approx_{i b}$. Also $\approx_{w h p b} \subsetneq \approx_{w h} \subsetneq \approx_{i b}$.

We give examples to show that the inclusions in Proposition 3.11 are proper. Here and subsequently we use a CCS-like notation to refer to simple configuration structures.

Example 3.12. IB equivalence is insensitive to auto-concurrency: $a \mid a=a$. $a$ holds for $\approx_{i b}$, but not for $\approx_{s b}$ neither for $\approx_{w h}$.

Example 3.13. $a \mid a=(a \mid a)+a . a$ holds for $\approx_{s b}$, but not for $\approx_{p b}$.

Example 3.14. (van Glabbeek and Goltz 2001, Ex 9.1) $a .(b+c)+(a \mid b)+a . b=$ $a .(b+c)+(a \mid b)$ holds for $\approx_{p b}$, but not for $\approx_{w h}$.

Example 3.15. (Bednarczyk 1991, Ex 1.6)(van Glabbeek and Goltz 2001, Ex 9.3) $\mathcal{E}$ and $\mathcal{F}$ are defined as in Figure 2. Recall that we depict the causal relation between events with arrows, and the conflict relation with dotted lines. $\mathcal{E}=\mathcal{F}$ holds for $\approx_{w h}$, but not for $\approx_{p b}$.

Example 3.16. (van Glabbeek and Goltz 2001, Ex $9.4(\mathrm{p} 294)) \mathcal{E}$ and $\mathcal{F}$ are defined as in Figure 3. $\mathcal{E}=\mathcal{F}$ holds for $\approx_{w h p b}$, but not for $\approx_{h}$.

Proposition 3.11 resolves all implications between the equivalences introduced so far, apart from the relationship between $\approx_{w h}$ and $\approx_{s b}$, which was resolved by Fecher: 

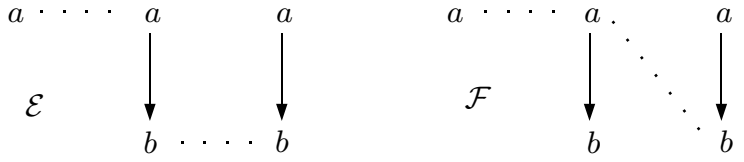

Fig. 2. Example 3.15
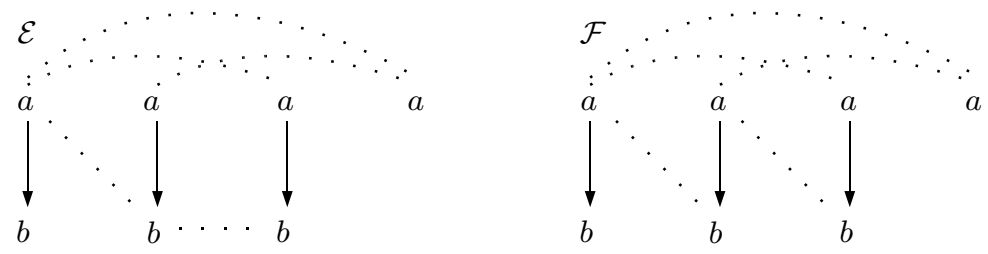

Fig. 3. Example 3.16

Proposition 3.17. (Fecher 2004, Theorem 9) On stable configuration structures, $\approx_{w h} \subseteq$ $\approx_{s b}$.

The inclusion in Proposition 3.17 is proper, since we know that $\approx_{s b} \nsubseteq \approx_{w h}$ by Example 3.14 .

\subsection{Depth-respecting equivalences}

We now use depth of an event within a configuration, i.e. the length of the longest causal chain leading up to and including the event, as a new notion of observation which we can make of an event, and study equivalences based on this notion. It will allow us to give a much shorter and more straightforward proof of Proposition 3.17, and will be the vital technique in proving results about reverse step equivalences in Section 5.

We start by defining depth:

Definition 3.18. Let $\mathcal{C}=(C, \ell)$ be a stable configuration structure, and let $X \in C$, $e \in X$. The depth of $e$ w.r.t. $X$ (and implicitly $\mathcal{C}$ ) is given by

$$
\operatorname{depth}_{X}(e) \stackrel{\text { df }}{=} \begin{cases}1 & \text { if } e \text { is minimal in } X \\ \max \left\{\operatorname{depth}_{X}\left(e^{\prime}\right): e^{\prime}<_{X} e\right\}+1 & \text { otherwise }\end{cases}
$$

The depth of an event $e$ is the length of the longest causal chain in $X$ up to and including $e$. Clearly, if $e<_{X} e^{\prime}$ then $\operatorname{depth}_{X}(e)<\operatorname{depth}_{X}\left(e^{\prime}\right)$. Note that if $X, Y \in C$ and $e \in X \cap Y$, then it is not necessarily the case that $\operatorname{depth}_{X}(e)=\operatorname{depth}_{Y}(e)$ (due to the fact that a single event can have different possible sets of causes). However if $X \cup Y \subseteq Z$ for some $Z \in C$ then $\operatorname{depth}_{X}(e)=\operatorname{depth}_{Y}(e)$.

Definition 3.19. Let $\mathcal{C}=(C, \ell)$ be a stable configuration structure and let $a \in$ Act, $k \in \mathbb{N}$. We let $X \stackrel{a, k}{\rightarrow} \mathcal{C} X^{\prime}$ iff $X, X^{\prime} \in C, X \subseteq X^{\prime}$ and $X^{\prime} \backslash X=\{e\}$ with $\ell(e)=a$, $\operatorname{depth}_{X^{\prime}}(e)=k$.

Definition 3.20. Let $\mathcal{C}, \mathcal{D}$ be stable configuration structures. A relation $R \subseteq C_{\mathcal{C}} \times C_{\mathcal{D}}$ 

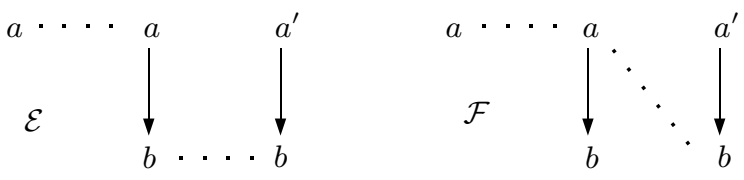

Fig. 4. Example 3.22.

is a depth-respecting bisimulation (DB) between $\mathcal{C}$ and $\mathcal{D}$ if $(\emptyset, \emptyset) \in R$ and if $(X, Y) \in R$ then for $a \in$ Act and $k \in \mathbb{N}$

— if $X \stackrel{a, k}{\rightarrow} \mathcal{C} X^{\prime}$ then $\exists Y^{\prime} . Y \stackrel{a, k}{\rightarrow} Y_{\mathcal{D}}^{\prime}$ and $\left(X^{\prime}, Y^{\prime}\right) \in R$;

- if $Y \stackrel{a, k}{\rightarrow} \mathcal{D} Y^{\prime}$ then $\exists X^{\prime} . X \stackrel{a, k}{\rightarrow} \mathcal{C} X^{\prime}$ and $\left(X^{\prime}, Y^{\prime}\right) \in R$.

We say that $\mathcal{C}$ and $\mathcal{D}$ are $\mathrm{DB}$ equivalent $\left(\mathcal{C} \approx_{d b} \mathcal{D}\right)$ iff there is a DB between $\mathcal{C}$ and $\mathcal{D}$.

Proposition 3.21. On stable configuration structures, $\approx_{d b} \subsetneq \approx_{s b}$.

Proof. Suppose that $\mathcal{C} \approx_{d b} \mathcal{D}$ via $\mathrm{DB} R$. We show that $R$ is an SB. Let $A \in \mathbb{N}^{A c t}$, and suppose $R(X, Y)$.

Assume $X \stackrel{A}{\rightarrow} \mathcal{C} X^{\prime}$. Let $E=X^{\prime} \backslash X$ and let $\left\{e_{1}, \ldots, e_{n}\right\}$ be an enumeration of $E$ in non-increasing order of depth w.r.t. $X^{\prime}$, i.e., letting $\operatorname{depth}_{X^{\prime}}\left(e_{i}\right)=k_{i}$ we have $k_{i} \geq k_{j}$ for $i<j \leq n$. Let $\ell_{\mathcal{C}}\left(e_{i}\right)=a_{i}(i \leq n)$. Then $X=X_{0} \stackrel{a_{1}, k_{1}}{\rightarrow} X_{1} \cdots \stackrel{a_{n}, k_{n}}{\rightarrow} X_{n}=X^{\prime}$. So $Y=Y_{0} \stackrel{a_{1}, k_{1}}{\rightarrow} Y_{1} \ldots \stackrel{a_{n}, k_{n}}{\rightarrow} Y_{n}=Y^{\prime}$ for some $Y_{1}, \ldots, Y_{n}$ such that $R\left(X_{i}, Y_{i}\right)(i \leq n)$. Let $e_{i}^{\prime}=Y_{i} \backslash Y_{i-1}(i=1, \ldots, n)$. The $e_{i}^{\prime}$ must all be pairwise concurrent: if $i<j$ then $e_{j}^{\prime}<_{Y^{\prime}} e_{i}^{\prime}$ is impossible since $e_{j}^{\prime} \notin Y_{i}$ and $Y_{i}$ is left-closed; also, $e_{i}^{\prime}<_{Y^{\prime}} e_{j}^{\prime}$ is impossible since depth ${ }_{Y^{\prime}}\left(e_{i}\right) \geq \operatorname{depth}_{Y^{\prime}}\left(e_{j}\right)$. Hence $Y \stackrel{A}{\rightarrow} \mathcal{D} Y^{\prime}$ with $R\left(X^{\prime}, Y^{\prime}\right)$, as required.

By symmetry, we also have that if $Y \stackrel{A}{\rightarrow} \mathcal{C} Y^{\prime}$ then $X \stackrel{A}{\rightarrow} \mathcal{C} X^{\prime}$ for some $X^{\prime}$ such that $R\left(X^{\prime}, Y^{\prime}\right)$. This shows that $\approx_{d b} \subseteq \approx_{s b}$.

The inclusion is proper by Example 3.13, since $a \mid a=(a \mid a)+a . a$ holds for $\approx_{s b}$, but not for $\approx_{d b}$.

Example 3.22. Modify (van Glabbeek and Goltz 2001, Example 9.3) by relabelling the last $a$ (reading from left to right) as $a^{\prime}$ to get $\mathcal{E}$ and $\mathcal{F}$ as in Figure 4 . This removes auto-concurrency. The modified structures are not PB-equivalent, since in the left-hand one, necessarily after $a$ we can always do pomset $a^{\prime}<b$, but not in the right-hand one. It is not hard to see that they are not WH-equivalent, either. Also, the modified structures are DB-equivalent.

The equivalences $\approx_{p b}$ and $\approx_{d b}$ are incomparable: $\approx_{d b} \nsubseteq \approx_{p b}$ by Example 3.22 , and $\approx_{p b} \nsubseteq \approx_{d b}$ by Example 3.14 , since this fails to hold for $\approx_{d b}$ as well as $\approx_{w h}$.

Lemma 3.23. Let $\mathcal{C}, \mathcal{D}$ be stable configuration structures. Let $X \in C_{\mathcal{C}}, Y \in C_{\mathcal{D}}$. Suppose $\left(X,<_{X}, \ell_{\mathcal{C}}\lceil X) \cong\left(Y,<_{Y}, \ell_{\mathcal{D}} \mid Y\right)\right.$ via isomorphism $f$. Then for any $e \in X, \operatorname{depth}_{X}(e)=$ $\operatorname{depth}_{Y}(f(e))$.

Proposition 3.24. On stable configuration structures, $\approx_{w h} \subsetneq \approx_{d b}$.

Proof. Suppose that $\mathcal{C} \approx_{d b} \mathcal{D}$ via WH bisimulation $R$. We show that $R$ is a DB. 


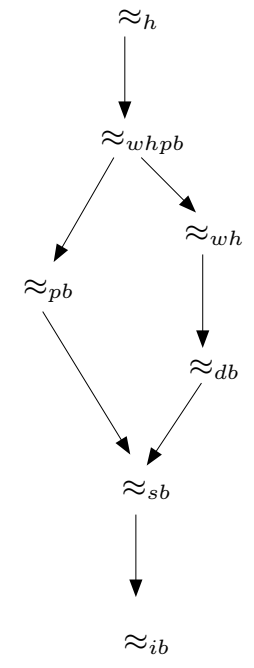

Fig. 5. Equivalences discussed so far.

Suppose that $R(X, Y)$ and $X \stackrel{a, k}{\rightarrow} \underset{C}{ } X^{\prime}$. Since $R$ is a WH bisimulation, there is $Y^{\prime}$ such that $Y \stackrel{a}{\rightarrow} \mathcal{C} Y^{\prime}$ and $R(X, Y)$. We know $X \cong Y$. Hence for each $k^{\prime} \geq 0, X$ and $Y$ have the same number of events with depth $k^{\prime}$, using Lemma 3.23. The same is true for $X^{\prime}$ and $Y^{\prime}$, since $X^{\prime} \cong Y^{\prime}$. Let $X^{\prime} \backslash X=\{e\}$ and $Y^{\prime} \backslash Y=\left\{e^{\prime}\right\}$. We have $\operatorname{depth}_{X^{\prime}}(e)=k$. Since $Y^{\prime}$ has one more event with depth $k$ than $Y$, and for any $k^{\prime} \neq k, Y^{\prime}$ has the same number of events of depth $k^{\prime}$ as $Y$, we deduce that $\operatorname{depth}_{Y^{\prime}}\left(e^{\prime}\right)=k$. Hence $Y \stackrel{a, k}{\rightarrow} Y^{\prime}$ as required.

The case when $Y \stackrel{a, k}{\rightarrow} \mathcal{D} Y^{\prime}$ is similar. The inclusion is proper by Example 3.22.

Combining Propositions 3.24 and 3.21 we obtain an alternative proof of Proposition 3.17 which is much shorter and more straightforward than the original proof in (Fecher 2004).

We show the inclusions between all equivalences introduced so far in Figure 5. All inclusions are proper, and no other inclusions hold, as we have seen by various counterexamples.

\subsection{Homogeneous and equidepth versions}

We define and discuss briefly three minor variants of step bisimulation. They are vital in the proofs of results in Section 5 and also have an impact on the developments in Section 9 where we improve on Bednarczyk's characterisation of H-H in the setting with no equidepth auto-concurrency.

Let us say that a set of events is homogeneous if all events have the same label. Similarly, a multiset of labels is homogeneous if all labels are the same. A step transition $X \stackrel{A}{\rightarrow} \mathcal{C} X^{\prime}$ is homogeneous if $A$ is homogeneous. We define homogeneous step bisimulation (HSB) and the associated equivalence $\approx_{h s b}$ by modifying step bisimulation (Definition 3.4) by requiring all step transitions to be homogeneous. 


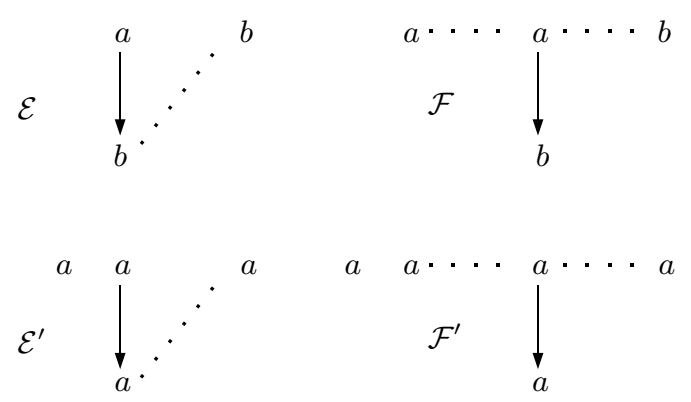

Fig. 6. Example 3.30.

We define the equidepth step transition relation:

Definition 3.25. Let $\mathcal{C}=(C, \ell)$ be a stable configuration structure and let $A \in \mathbb{N}^{A c t}$.

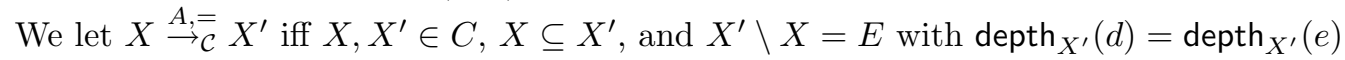
for all $d, e \in E$ and $\ell(E)=A$.

It is immediate that if $X \stackrel{A,=}{\rightarrow \mathcal{C}} X^{\prime}$ then $X \stackrel{A}{\rightarrow} \mathcal{C} X^{\prime}$ in the usual sense.

We can then define

- equidepth step bisimulation and $\approx_{e s b}$

- homogeneous equidepth step bisimulation and $\approx_{\text {hesb }}$

in an obvious fashion.

The equation $a \mid a=a . a$ (Example 3.12 ) holds for $\approx_{i b}$ but not for $\approx_{h s b}$ or $\approx_{h e s b}$.

Example 3.26. For $a \neq b: a \mid b=a . b+b . a$ holds for $\approx_{h s b}$ and $\approx_{h e s b}$, but not for $\approx_{s b}$ or $\approx_{e s b}$.

Example 3.27. $a \mid(b . a)=(a \mid b) . a($ where $a \neq b)$ holds for $\approx_{e s b}$, but not for $\approx_{h s b}$.

Proposition 3.28. On stable configuration structures,

$1 \approx_{d b} \subsetneq \approx_{e s b} \subsetneq \approx_{h e s b} \subsetneq \approx_{i b}$

$2 \approx_{s b} \subsetneq \approx_{h s b} \subsetneq \approx_{i b}$

Proof. It is straightforward to show the inclusions. They are proper by Examples 3.12, 3.26 and 3.27 .

The next example shows that $\approx_{s b} \nsubseteq \approx_{h e s b}$ :

Example 3.29. $a|(a . a)+a .(a \mid a)=a|(a . a)$ holds for $\approx_{s b}$, but not for $\approx_{h e s b}$.

Since Example 3.29 does not hold for $\approx_{p b}$, we need a different example to show that $\approx_{p b} \nsubseteq \approx_{h e s b}$.

Example 3.30. The first pair of structures $\mathcal{E}, \mathcal{F}$ in Figure 6 are PB- and ESB-equivalent, but not DB-equivalent. This example is taken from (van Glabbeek and Goltz 1989). We modify $\mathcal{E}, \mathcal{F}$ by letting all events have the same label $a$ and adding a new event in parallel, also labelled with $a$. This yields $\mathcal{E}^{\prime}, \mathcal{F}^{\prime}$ as shown also in Figure 6 . Let $R=\{(X, Y): X \in$ 


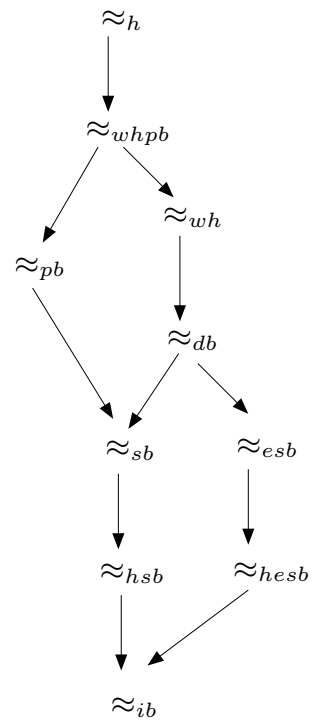

Fig. 7. Adding the homogeneous and equidepth step equivalences.

$\left.C_{\mathcal{E}^{\prime}}, Y \in C_{\mathcal{F}^{\prime}},|X|=|Y|\right\}$. It can be checked that $R$ is a $\mathrm{PB}$, so that $\mathcal{E}^{\prime} \approx_{p b} \mathcal{F}^{\prime}$. However $\mathcal{E}^{\prime} \not \varkappa_{\text {hesb }} \mathcal{F}^{\prime}$. This is because in $\mathcal{E}^{\prime}$, after performing $a$, an equidepth $\{a, a\}$ transition is always possible, while in $\mathcal{F}^{\prime}$ after performing the $a$ in conflict with two other events, no such equidepth $\{a, a\}$ transition is possible.

In Figure 7 we show the inclusions between all the equivalences introduced so far. All inclusions are proper. Any inclusions not shown do not hold, as demonstrated by the various examples we have presented.

\section{Reverse single-event transitions}

In this section we explore the hierarchy of equivalences where we add the power of reverse computation with single-event transitions.

We use a reverse transition relation, with a wavy arrow, which simply inverts the standard forward version.

Definition 4.1. Let $\mathcal{C}=(C, \ell)$ be a stable configuration structure and let $a \in$ Act. Then $X \stackrel{a}{\sim} \mathcal{C} X^{\prime}$ iff $X^{\prime} \stackrel{a}{\rightarrow} \mathcal{C} X$.

We can now define reverse single-event versions of all the equivalences defined in Section 3 . Let FB stand for any of the forward-only bisimulations $\approx_{f b}$ defined in Section 3 , apart from history-preserving $(\mathrm{H})$ bisimulation. Thus FB may be any one of IB, SB, PB, WH, WHPB, DB, etc.

Definition 4.2. Let $\mathcal{C}=(C, \ell)$ be a stable configuration structure and let $a \in$ Act. Then $R \subseteq C_{\mathcal{C}} \times C_{\mathcal{D}}$ is an RI-FB iff $R$ is an FB and if $R(X, Y)$ then for any $a \in$ Act, 
— if $X \stackrel{a}{\rightsquigarrow \mathcal{C}} X^{\prime}$ then $\exists Y^{\prime} . Y \stackrel{a}{\rightsquigarrow \mathcal{D}} Y^{\prime}$ and $\left(X^{\prime}, Y^{\prime}\right) \in R$;

- if $Y \stackrel{a}{\sim} \mathcal{D} Y^{\prime}$ then $\exists X^{\prime} . X \stackrel{a}{\sim} \mathfrak{C} X^{\prime}$ and $\left(X^{\prime}, Y^{\prime}\right) \in R$.

We say that $\mathcal{C}$ and $\mathcal{D}$ are RI-FB equivalent $\left(\mathcal{C} \approx_{r i-f b} \mathcal{D}\right)$ iff there is an RI-FB bisimulation between $\mathcal{C}$ and $\mathcal{D}$.

Definition 4.3. Let $\mathcal{C}=(C, \ell)$ be a stable configuration structures and let $a \in$ Act. Then $R \subseteq C_{\mathcal{C}} \times C_{\mathcal{D}} \times \mathcal{P}\left(E_{\mathcal{C}} \times E_{\mathcal{D}}\right)$ is an RI-H bisimulation iff $R$ is an $\mathrm{H}$ bisimulation and if $R(X, Y, f)$ then for any $a \in$ Act,

— if $X \stackrel{a}{\sim} \mathcal{C} X^{\prime}$ then $\exists Y^{\prime}, f^{\prime} . Y \stackrel{a}{\sim} \mathcal{D} Y^{\prime}$ and $\left(X^{\prime}, Y^{\prime}, f^{\prime}\right) \in R$;

— if $Y \stackrel{a}{\sim} \mathcal{D} Y^{\prime}$ then $\exists X^{\prime}, f^{\prime} . X \stackrel{a}{\sim} \mathcal{C} X^{\prime}$ and $\left(X^{\prime}, Y^{\prime}, f^{\prime}\right) \in R$.

We say that $\mathcal{C}$ and $\mathcal{D}$ are RI-H equivalent $\left(\mathcal{C} \approx_{r i-h} \mathcal{D}\right)$ iff there is an RI-H bisimulation between $\mathcal{C}$ and $\mathcal{D}$.

Notice that in Definition 4.3 we do not assert any relationship between $f^{\prime}$ and $f$.

Remark 4.4. What we here call $\approx_{r i-i b}$ was defined in (Bednarczyk 1991), where it is called back $\&$ forth bisimulation $\left(\sim_{b \& f}\right)$. It was called forward-reverse $(\mathrm{FR})$ bisimulation in (Phillips and Ulidowski 2007), and reverse bisimulation (RB) in (Phillips and Ulidowski 2010).

De Nicola, Montanari and Vaandrager also investigated "back \& forth" bisimulations (De Nicola et al. 1990; De Nicola and Vaandrager 1990), but their relations were defined over computations (paths) rather than states (for example, in the process $a \mid b$, after performing $a$ followed by $b$, one can only reverse immediately on $b$, and not $a$ ). As a result, in the absence of $\tau$ actions, the distinguishing power of these bisimulations is that of IB (De Nicola et al. 1990); hence, it is lower than that of RI-IB.

As far as we are aware, $\approx_{r i-f b}$ equivalences other than $\approx_{r i-i b}$ have not been investigated previously.

Given any two forward-only equivalences $\approx_{f b}, \approx_{f b^{\prime}}$, it is clear from the definitions that if $\approx_{f b} \subseteq \approx_{f b^{\prime}}$ then $\approx_{r i-f b} \subseteq \approx_{r i-f b^{\prime}}$. It is also immediate from the definitions that for any forward-only equivalence $\approx_{f b}$ we have $\approx_{r i-f b} \subseteq \approx_{f b}$.

In Figure 8 we show the inclusions between all the $\approx_{r i-f b}$ equivalences. We now give various examples to show that the inclusions denoted by solid arrows are proper:

- The equation $a \mid a=a . a$ (Example 3.12 ) holds for $\approx_{r i-i b}$ but not for $\approx_{s b}$, and therefore not for $\approx_{r i-s b}$.

- The equation $a \mid a=(a \mid a)+a . a$ (Example 3.13) holds for $\approx_{r i-s b}$, but not for $\approx_{p b}$ or $\approx_{d b}$, and therefore not for $\approx_{r i-p b}$ or $\approx_{r i-d b}$.

Both examples just cited use auto-concurrency, and we see that the extra power of reverse single-event transitions has made no difference in these two cases.

When it comes to distinguishing $\approx_{r i-h}, \approx_{r i-w h p b}, \approx_{r i-w h}$ and $\approx_{r i-p b}$ we cannot use Examples 3.14, 3.15 and 3.16, as we did in the forward-only case, since all three examples are invalid for $\approx_{r i-i b}$. We have not been able to locate any examples in the literature which would serve the purpose, and so we present three new examples (Examples 4.5, 4.7 and 4.8$)$. 


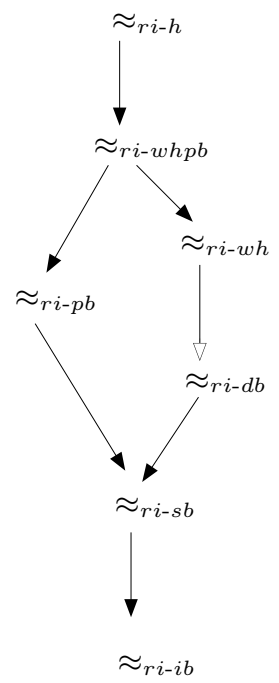

Fig. 8. Equivalences with reverse single-event transitions. Solid arrows represent proper inclusions, while the open arrow represents an inclusion not known to be proper.
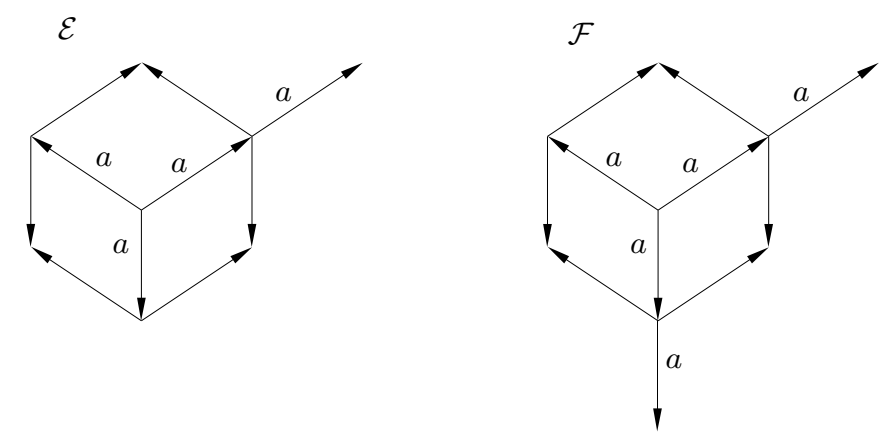

Fig. 9. Example 4.5.

Example 4.5. Figure 9 shows the transition systems for structures $\mathcal{E}, \mathcal{F}$. $\mathcal{E}=\mathcal{F}$ holds for $\approx_{r i-p b}$, but not for $\approx_{r i-d b}$, and hence not for $\approx_{r i-w h}$. Notice that this example uses non-binary conflict between the three initial $a$-events.

As a simpler alternative to Example 4.5 we have:

Example 4.6. Figure 10 shows the transition systems for structures $\mathcal{E}, \mathcal{F}$. $\mathcal{E}=\mathcal{F}$ holds for $\approx_{r i-p b}$, but not for $\approx_{d b}$, and hence not for $\approx_{r i-d b}$ or $\approx_{r i-w h}$.

Example 4.7. We adapt Example 3.15 (which was suggested to van Glabbeek \& Goltz by Rabinovich) by adding a new event $\left(b_{1}\right)$, to obtain the structures $\mathcal{E}, \mathcal{F}$ shown in Figure 11. Here as elsewhere, when we label events as $a_{1}, a_{2}, \ldots$ we mean that there are distinct events $e_{1}, e_{2}, \ldots$ which are labelled with $a$. Then $\mathcal{E} \not_{p b} \mathcal{F}$, by the same reasoning 

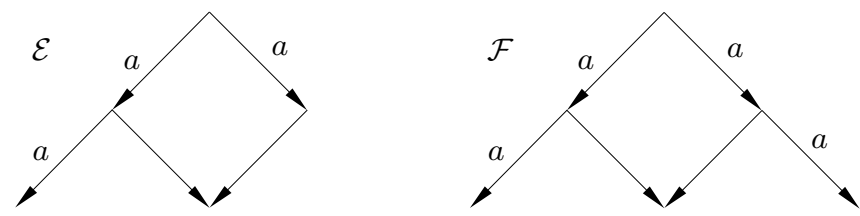

Fig. 10. Example 4.6.
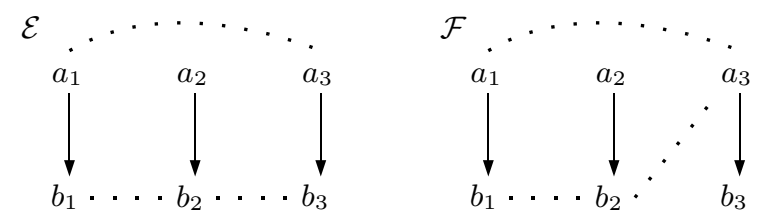

Fig. 11. Example 4.7.

as in (van Glabbeek and Goltz 2001): in $\mathcal{E}$, after any a can always perform pomset transition $a \rightarrow b$, whereas in $\mathcal{F}$, after $a_{3}$ cannot perform $a \rightarrow b$; see Figure 12 .

We now check that $\mathcal{E} \approx_{r i-w h} \mathcal{F}$. The only difference is that configuration $13\left(\left\{a_{2}, a_{3}, b_{3}\right\}\right)$ is missing in $\mathcal{F}$. We define $R=\left\{(X, Y): X \in C_{\mathcal{E}}, Y \in C_{\mathcal{F}}, X \cong Y\right\}$ and check that $R$ is an RI-WH bisimulation. See the transition diagram in Figure 13. The meaning is that all configurations in each source set can perform a transition to at least one member of the target set. Also, each configuration in the target set can perform a reverse transition to at least one member of the source set. This works for both $\mathcal{E}$ and $\mathcal{F}$, showing that $\mathcal{E} \approx_{\text {ri-wh }} \mathcal{F}$.

Example 4.8. We adapt Example 3.16 by adding a new event $\left(b_{4}\right)$ and changing the conflict between $a_{1}$ and $b_{2}$ to between $b_{1}$ and $b_{2}$, to obtain the structures $\mathcal{E}, \mathcal{F}$ shown in Figure $14 . \mathcal{E}=\mathcal{F}$ holds for $\approx_{r i-w h p b}$, but not for $\approx_{h}$, and hence not for $\approx_{r i-h}$. We now check that $\mathcal{E} \approx_{r i-w h p b} \mathcal{F}$. We give transition diagrams in Figure 15 . The only difference is that configuration $18\left(\left\{a_{2}, a_{3}, b_{3}\right\}\right)$ is missing in $\mathcal{F}$. We define a bisimulation by relating
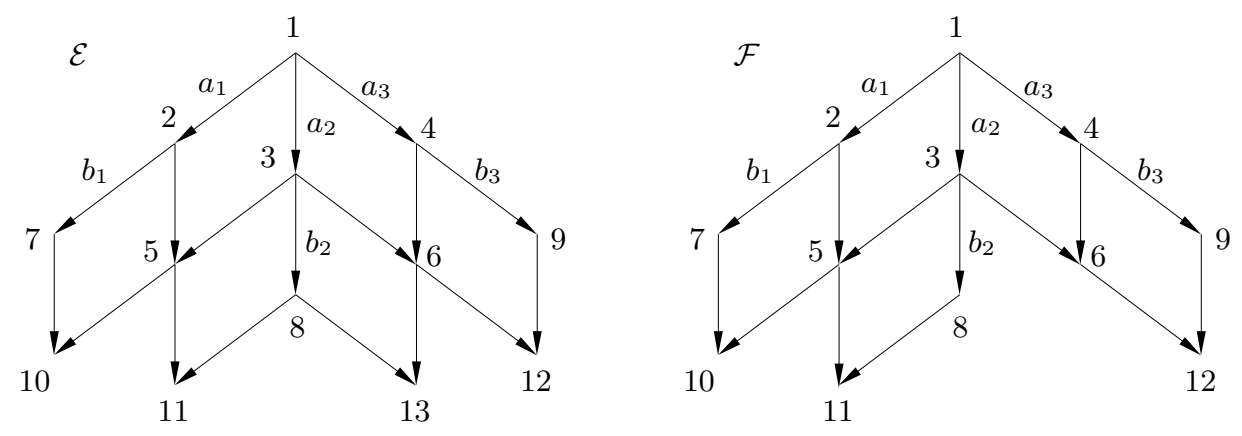

Fig. 12. Transition diagram for Example 4.7. 


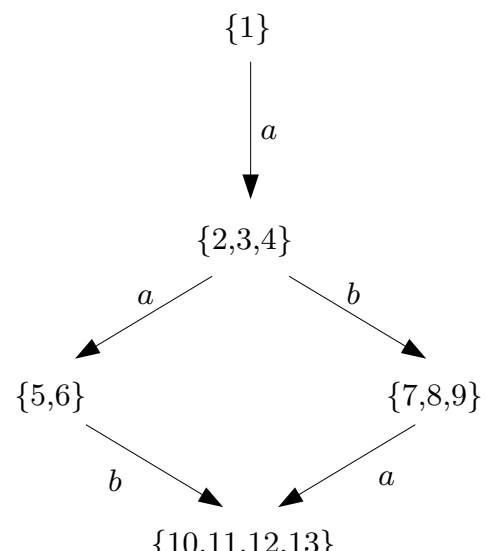

Fig. 13. Bisimulation for Example 4.7.
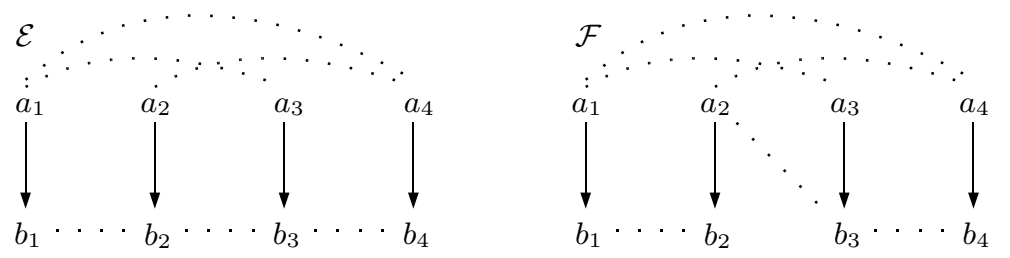

Fig. 14. Example 4.8.
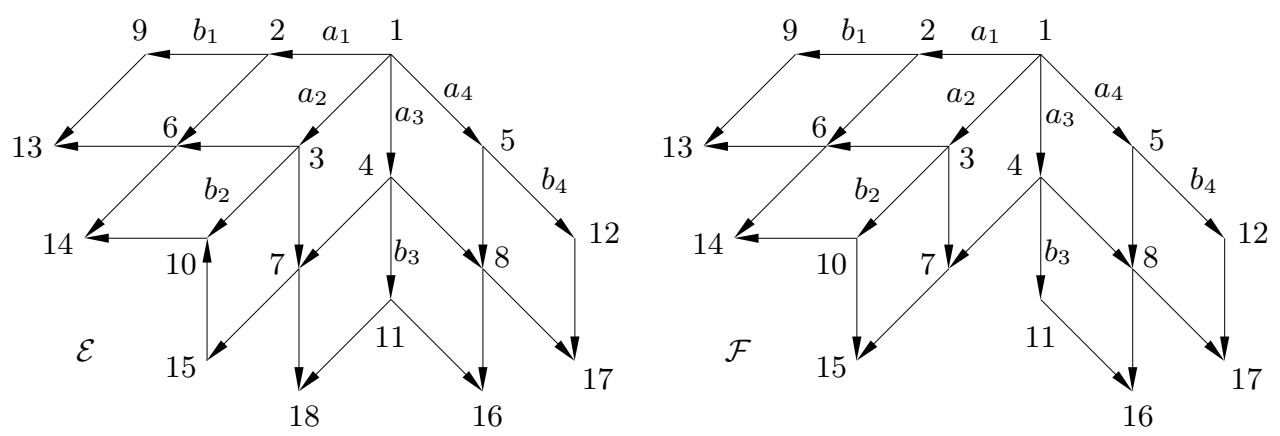

Fig. 15. Transition diagram for Example 4.8. 


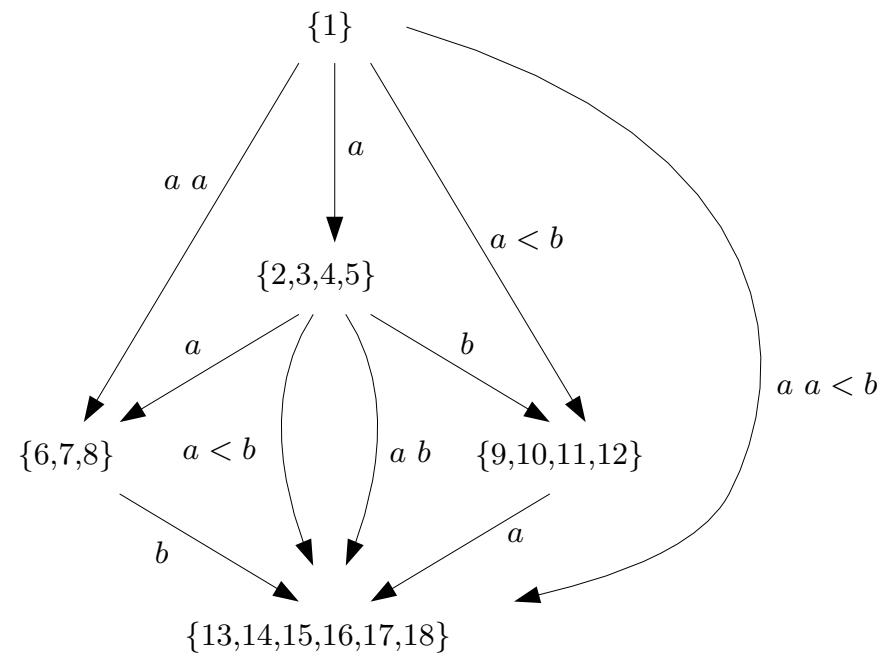

Fig. 16. Bisimulation for Example 4.8.

all isomorphic states, and check that it is an RI-WHPB; see the transition diagram in Figure 16. All pomset transitions work equally well for $\mathcal{E}$ and $\mathcal{F}$, in both directions.

To see that $\mathcal{E}$ and $\mathcal{F}$ are not $\mathrm{H}$-equivalent, consider $1 \stackrel{a_{2}}{\rightarrow} \rightarrow$ matched by $1 \stackrel{a_{i}}{\longrightarrow} \stackrel{a_{j}}{\longrightarrow}\left\{a_{i}, a_{j}\right\}$ in $\mathcal{E}$. Here $\left\{a_{i}, a_{j}\right\}$ must be one of 6,7 or 8 . But then both $b_{i}$ and $b_{j}$ are possible. However 7 in $\mathcal{F}$ can only do $b_{2}$. Hence one of the $b_{i}$ and $b_{j}$ transitions in $\mathcal{E}$ cannot be matched. (We have to stick to the isomorphism already established for $a_{2}, a_{3}$ to be history-preserving.)

We have now shown that all the inclusions in Figure 8 represented by solid arrows are proper. There is one open arrow from $\approx_{r i-w h}$ to $\approx_{r i-d b} ;$ it is an open question whether this inclusion is proper. All other inclusions are ruled out by the various examples we have presented.

We now turn to the question of relationships between the forward-only equivalences of Figure 7 and the reverse single-event versions of Figure 8. We have already seen that for every forward-only equivalence $\mathrm{FB}, \approx_{r i-f b} \subsetneq \approx_{f b}$. These latter inclusions are all proper as shown by the next example, which shows that the simplest reverse equivalence $\approx_{r i-i b}$ has extra power not available to the strongest forward-only equivalence $\approx_{h}$ :

Example 4.9. The Absorption Law (Boudol and Castellani 1987; Bednarczyk 1991; van Glabbeek and Goltz 2001)

$$
(a \mid(b+c))+(a \mid b)+((a+c) \mid b)=(a \mid(b+c))+((a+c) \mid b)
$$

holds for $\approx_{h}$, but not for $\approx_{r i-i b}$.

Another example which holds for $\approx_{h}$, but not for $\approx_{r i-i b}$ appears in (Nielsen and Clausen 1994, Example 1).

We now show that no other inclusions from the reverse single-event equivalences to 

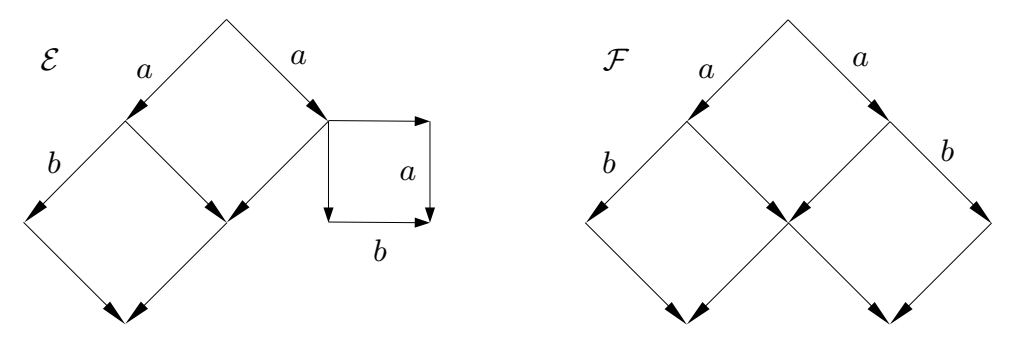

Fig. 17. An example where $\mathcal{E} \approx_{\text {ri-hesb }} \mathcal{F}$ but $\mathcal{E} \not_{\text {ri-esb }} \mathcal{F}$.

the forward-only equivalences are possible, apart from possibly $\approx_{r i-d b} \subsetneq \approx_{w h}$. This can be done using the examples already introduced:

$-\approx_{r i-w h p b} \not \subset \approx_{h}$ by Example 4.8 ;

- One can check that in Example 3.30, $\mathcal{E}^{\prime} \approx_{r i-p b} \mathcal{F}^{\prime}$ using the same bisimulation $R$ already given. Hence $\approx_{r i-p b} \nsubseteq \approx_{\text {hesb }}$;

$-\approx_{r i-w h} \not \subset \approx_{p b}$ by Example 4.7 ;

— $\approx_{r i-i b} \nsubseteq \approx_{h s b}, \approx_{r i-i b} \nsubseteq \approx_{h e s b}$ by Example 3.12 .

Remark 4.10. We could also consider reverse single-event versions of $\approx_{h s b}, \approx_{e s b}$ and $\approx_{h e s b}$. Example 3.12 holds for $\approx_{r i-i b}$, and fails for $\approx_{h s b}$ and $\approx_{h e s b}$, as we have already seen. Therefore $\approx_{r i-h s b}$ and $\approx_{r i-h e s b}$ are strictly included in $\approx_{r i-i b}$. Furthermore Example 3.13 holds for $\approx_{r i-e s b}$, and fails for $\approx_{d b}$. Hence $\approx_{r i-d b}$ is strictly included in $\approx_{\text {ri-esb }}$. A different example shows that $\approx_{r i-e s b}$ is strictly included in $\approx_{r i-h e s b}$ - see Figure 17 . However open questions remain, in particular whether $\approx_{r i-s b}$ is more powerful than $\approx_{r i-h s b}$.

\section{Reverse steps and depth-preserving transitions}

In this section we prove results about reverse step equivalences and reverse depthpreserving equivalences. We show in Section 5.1 that reverse steps are strictly more discriminating than forward steps. In Section 5.2 we show that reverse depth-preserving equivalences have the same power as reverse step equivalences. The technical development needed to show this will also be needed in Section 9. In Section 5.3 we put together the results of Sections 5.1 and 5.2, and map out the resulting hierarchy of equivalences.

\subsection{Reverse step equivalences}

As with single events, we use a reverse step transition relation, with a wavy arrow, which simply inverts the standard forward step version.

Definition 5.1. Let $\mathcal{C}=(C, \ell)$ be a stable configuration structure and let $A \in \mathbb{N}^{\text {Act. }}$. Then $X \stackrel{A}{\sim} \mathcal{C} X^{\prime}$ iff $X^{\prime} \stackrel{A}{\rightarrow} \mathcal{C} X$.

Much as for single event reverse transitions, we can now define reverse step versions $\approx_{r s-f b}$ and $\approx_{r s-h}$ of all the equivalences defined in Section 3. We omit the definitions, 
which are similar to Definitions 4.2 and 4.3 . We can define equivalences $\approx_{r h s-f b}$ in a corresponding way.

Remark 5.2. What we here call $\approx_{r s-s b}$ was briefly mentioned in (Bednarczyk 1991), where it is called multi-step back $\&$ forth bisimulation $\left(\sim_{\mu b \& f}\right)$. It was called reverse step bisimulation (RSB) in (Phillips and Ulidowski 2010).

Recall the notion of homogeneous forward step from Section 3.3. There we saw that homogeneous forward steps give less power than full forward steps: $\approx_{h s b} \subsetneq \approx_{s b}$. We now show that reverse homogeneous steps give as much power as full reverse steps, and that in fact they also have the power of forward steps: we prove $\approx_{r h s-i b}=\approx_{r s-s b}$ (Theorem 5.10).

We have seen that $a \mid a=(a \mid a)+a . a$ (Example 3.13$)$ holds for $\approx_{r i-s b}$. However it does not hold for $\approx_{r s-i b}$. Using Theorem 5.10 , this means that $\approx_{r i-s b}$ is strictly weaker than $\approx_{r s-i b}$. Thus reverse steps have greater power than forward steps.

Clearly, any RS-SB is an RHS-SB. We shall show the converse, so that $\approx_{r h s-i b}=\approx_{r s-s b}$ (Theorem 5.10).

First we need some lemmas.

Lemma 5.3. Let $\mathcal{C}$ and $\mathcal{D}$ be stable configuration structures and let $R$ be an RI-IB between $\mathcal{C}$ and $\mathcal{D}$. If $R(X, Y)$ then $\ell(X)=\ell(Y)$.

Proof. Suppose that $R(X, Y)$ and suppose that $\ell(X)=A$. By connectedness, there are transitions $X \stackrel{a_{1}}{\sim} \mathcal{C} \cdots \stackrel{a_{n}}{\sim} \mathcal{C} \emptyset$ with $A=\left\{a_{1}, \ldots, a_{n}\right\}$. Therefore $Y \stackrel{a_{1}}{\rightsquigarrow} \mathcal{D} \cdots \stackrel{a_{n}}{\rightsquigarrow} \mathcal{D} Y^{\prime}$, for some $Y^{\prime}$. Hence $\ell(X) \subseteq \ell(Y)$. Symmetrically, $\ell(Y) \subseteq \ell(X)$.

Note that Lemma 5.3 would not hold for IBs (or indeed SBs or PBs): for instance, there is an IB between $a+b$ and itself which, while necessarily including the identity relation on configurations, also relates the configuration resulting after performing (the event labelled) $a$ with that after performing $b$.

For any configuration $X$, let $\min (X)$ denote its set of minimal elements (w.r.t. $<_{X}$ ); $\min (X)$ is, of course, also a configuration, since it is a left-closed subset of $X$. Note that if $X, Y$ are configurations and $X \subseteq Y$ then $\min (X) \subseteq \min (Y)$.

Lemma 5.4. Let stable configuration structures $\mathcal{C}, \mathcal{D}$ be related by RHS-IB $R$. If $R(X, Y)$ then we have $R(\min (X), \min (Y))$.

Proof. Suppose $R(X, Y)$. Then there are $a_{1}, \ldots, a_{n}$ such that $X \stackrel{a_{1}}{\rightsquigarrow} \ldots \stackrel{a_{n}}{\rightsquigarrow} \min (X)$. Let $Y^{\prime}$ be such that $Y \stackrel{a_{1}}{\rightsquigarrow} \ldots \stackrel{a_{n}}{\rightsquigarrow} Y^{\prime}$ and $R\left(\min (X), Y^{\prime}\right)$. Then $\ell(\min (X))=\ell\left(Y^{\prime}\right)$ by Lemma 5.3. We show that $Y^{\prime}=\min (Y)$.

Now we use the reverse homogeneous steps. Let $A=\ell(\min (X))$. Take any $a \in A$, and let $A_{a}$ be the multiset of $a$ s in $A$. Then $\min (X) \stackrel{b_{1}}{\rightsquigarrow} \ldots \stackrel{b_{n}}{A_{a}} \rightsquigarrow \emptyset$ for some $b_{1}, \ldots, b_{n} \neq a$. Hence $Y^{\prime} \stackrel{b_{1}}{\rightsquigarrow \ldots \stackrel{b_{n}}{\rightsquigarrow} A_{a}} \emptyset$. This tells us that all events labelled with $a$ in $Y^{\prime}$ are minimal. Hence all events in $Y^{\prime}$ are minimal, since $a$ was arbitrary. So $Y^{\prime} \subseteq \min (Y)$. Hence $\ell(\min (X)) \subseteq \ell(\min (Y))$. Symmetrically we can establish $\ell(\min (Y)) \subseteq \ell(\min (X))$. So $\ell(\min (Y))=\ell(\min (X))$. Hence $Y^{\prime}=\min (Y)$ and $R(\min (X), \min (Y))$ as required. 
We now define the "lifting" of a configuration structure with respect to a configuration $M$ :

Definition 5.5. Let $\mathcal{C}=(C, \ell)$ be a stable configuration structure and let $M \in C$. Define $\mathcal{C}_{M}=\left(C_{M}, \ell_{M}\right)$ where $C_{M}=\{X \backslash M: M \subseteq X \in C, \min (X)=\min (M)\}$ and $\ell_{M}=\ell \uparrow \bigcup_{Y \in C_{M}} Y$.

Lemma 5.6. Let $\mathcal{C}=(C, \ell)$ be a stable configuration structure and let $M \in C$. Then $\mathcal{C}_{M}$ is a stable configuration structure.

Lemma 5.7. Let stable configuration structures $\mathcal{C}, \mathcal{D}$ be related by RHS-IB $R$. Let $M \in C_{\mathcal{C}}$ be such that $\min (M)=M$. Similarly, let $N \in C_{\mathcal{D}}$ be such that $\min (N)=N$. Suppose also that $R(M, N)$. Define $R_{M, N}$ by

$$
R_{M, N}=\{(X \backslash M, Y \backslash N): R(X, Y), \min (X)=M, \min (Y)=N\}
$$

Then $R_{M, N}$ is an RHS-IB between $\mathcal{C}_{M}$ and $\mathcal{D}_{N}$.

Proof. Write $R_{M, N}$ as $R^{\prime}$ for short. We certainly have $R^{\prime}(\emptyset, \emptyset)$, since $R(M, N)$. Suppose $R^{\prime}(X \backslash M, Y \backslash N)$ with $R(X, Y), \min (X)=M, \min (Y)=N$.

Forwards: Suppose $X \backslash M \stackrel{a}{\rightarrow} \mathcal{C}_{M} X^{\prime} \backslash M$, where $X^{\prime} \in C_{\mathcal{C}}$ and $\min \left(X^{\prime}\right)=M$. Then $X \stackrel{a}{\rightarrow} \mathcal{C} X^{\prime}$. Hence there is $Y^{\prime}$ such that $Y \stackrel{a}{\rightarrow} \mathcal{D} Y^{\prime}$ and $R\left(X^{\prime}, Y^{\prime}\right)$. We need to know that $\min \left(Y^{\prime}\right)=N$. Using Lemma 5.4 we have $R\left(M, \min \left(Y^{\prime}\right)\right)$. By Lemma 5.3 , since $R(M, N)$ we have $\ell(M)=\ell(N)$. Also since $R\left(M, \min \left(Y^{\prime}\right)\right)$ we have $\ell(M)=\ell\left(\min \left(Y^{\prime}\right)\right)$. So $\ell(N)=\ell\left(\min \left(Y^{\prime}\right)\right)$. Since $N=\min (Y) \subseteq \min \left(Y^{\prime}\right)$ we deduce $\min \left(Y^{\prime}\right)=N$. Now we obtain $Y \backslash N \stackrel{a}{\rightarrow} \mathcal{D}_{N} Y^{\prime} \backslash N$ and $R^{\prime}\left(X^{\prime} \backslash M, Y^{\prime} \backslash N\right)$ as required.

Reverse: Suppose $X \backslash M \stackrel{A}{\sim} \mathcal{C}_{M} X^{\prime} \backslash M$, where $X^{\prime} \in C_{\mathcal{C}}$ and $\min \left(X^{\prime}\right)=M$. Then $X \stackrel{A}{\sim} \mathcal{C} X^{\prime}$. Hence there is $Y^{\prime}$ such that $Y \stackrel{A}{{ }_{m}} \mathcal{D} Y^{\prime}$ and $R\left(X^{\prime}, Y^{\prime}\right)$. We need to know that $\min \left(Y^{\prime}\right)=N$. By Lemma 5.4 we have $R\left(M, \min \left(Y^{\prime}\right)\right)$. So by Lemma $5.3, \ell\left(\min \left(Y^{\prime}\right)\right)=$ $\ell(M)=\ell(N)$. Since $Y^{\prime} \subseteq Y, \min \left(Y^{\prime}\right) \subseteq \min (Y)=N$. Hence $\min \left(Y^{\prime}\right)=N$. Now we get $Y \backslash N \stackrel{A}{\sim} \mathcal{D}_{N} Y^{\prime} \backslash N$ and $R^{\prime}\left(X^{\prime} \backslash M, Y^{\prime} \backslash N\right)$ as required.

Lemma 5.8. Let stable configuration structures $\mathcal{C}, \mathcal{D}$ be related by RHS-IB $R$. Suppose $R(X, Y)$ and $X \stackrel{A}{\rightarrow} \mathcal{C} X^{\prime}$. Then there is $Y^{\prime}$ such that $Y \stackrel{A}{\rightarrow} \mathcal{D} Y^{\prime}$ and $R\left(X^{\prime}, Y^{\prime}\right)$.

Proof. The proof is partially inspired by Fecher's proof that a weak history-preserving bisimulation is a step bisimulation (Fecher 2004).

We proceed by induction on $\left|X^{\prime}\right|$.

Base case: $\left|X^{\prime}\right|=0$. Then $Y^{\prime}=\emptyset$ will do, trivially.

Induction step. Notice that $\min (X) \subseteq \min \left(X^{\prime}\right)$. There are two cases:

(1) There is $e \in \min \left(X^{\prime}\right) \backslash \min (X)$. Let $\ell(e)=a$. Then $X \stackrel{A \backslash\{a\}}{\longrightarrow} X^{\prime} \backslash\{e\}$. By induction there is $Y^{\prime \prime}$ such that $Y^{A} \stackrel{A \backslash a\}}{\longrightarrow} Y^{\prime \prime}$ and $R\left(X^{\prime} \backslash\{e\}, Y^{\prime \prime}\right)$. Now $X^{\prime} \backslash\{e\} \stackrel{a}{\rightarrow} X^{\prime}$. So there is $Y^{\prime}$ such that $Y^{\prime \prime} \stackrel{a}{\rightarrow} Y^{\prime}$ and $R\left(X^{\prime}, Y^{\prime}\right)$. Let $e^{\prime}$ be the single element in $Y^{\prime} \backslash Y^{\prime \prime}$. Now $\min \left(X^{\prime} \backslash\{e\}\right)=\min \left(X^{\prime}\right) \backslash\{e\}\left(\right.$ and $\left.e \in \min \left(X^{\prime}\right)\right)$. So $\left|\min \left(X^{\prime} \backslash\{e\}\right)\right|<\left|\min \left(X^{\prime}\right)\right|$. By Lemmas 5.4 and 5.3, $\ell\left(\min \left(X^{\prime} \backslash\{e\}\right)\right)=\ell\left(\min \left(Y^{\prime} \backslash\left\{e^{\prime}\right\}\right)\right)$ and $\ell\left(\min \left(X^{\prime}\right)\right)=\ell\left(\min \left(Y^{\prime}\right)\right)$. 
So $\left|\min \left(Y^{\prime} \backslash\left\{e^{\prime}\right\}\right)\right|<\left|\min \left(Y^{\prime}\right)\right|$ and $\min \left(Y^{\prime} \backslash\left\{e^{\prime}\right\}\right) \subsetneq \min \left(Y^{\prime}\right)$. It follows that $e^{\prime} \in \min \left(Y^{\prime}\right)$. Hence $e^{\prime}$ is concurrent with all events in $Y^{\prime} \backslash Y$, and $Y \stackrel{A}{\rightarrow} Y^{\prime}$ as required.

(2) $\min \left(X^{\prime}\right)=\min (X)$. Let $M=\min (X), N=\min (Y)$. By Lemma 5.4 we have $R(M, N)$.

Let configuration structures $\mathcal{C}_{M}$ and $\mathcal{D}_{N}$ be as in Definition 5.5. Let $R^{\prime}$ be the RHS-IB between $\mathcal{C}_{M}$ and $\mathcal{D}_{N}$ of Lemma 5.7.

We have $R^{\prime}(X \backslash M, Y \backslash N)$ and $X \backslash M \stackrel{A}{\rightarrow} \mathcal{C}_{M} X^{\prime} \backslash M$. Clearly $M \neq \emptyset$, since $\left|X^{\prime}\right|>0$. So by induction there is $Y^{\prime}$ such that $Y \backslash N \stackrel{A}{\rightarrow} \mathcal{D}_{N} Y^{\prime} \backslash N$ with $R^{\prime}\left(X^{\prime} \backslash M, Y^{\prime} \backslash N\right)$. So $Y \stackrel{A}{\rightarrow}_{\mathcal{D}} Y^{\prime}$ and $R\left(X^{\prime}, Y^{\prime}\right)$ as required.

We use the same method as Lemma 5.8 to show:

Lemma 5.9. Let stable configuration structures $\mathcal{C}, \mathcal{D}$ be related by RHS-IB $R$. Suppose $R(X, Y)$ and $X \stackrel{A}{\sim} \mathcal{C} X^{\prime}$. Then there is $Y^{\prime}$ such that $Y \stackrel{A}{\sim} \mathcal{D} Y^{\prime}$ and $R\left(X^{\prime}, Y^{\prime}\right)$.

Proof. Much as the proof of Lemma 5.8. We proceed by induction on $|X|$, going into much the same two cases.

In (1) note that we start by reversing from $X$ in a single event transition using an element $e \in \min (X) \backslash \min \left(X^{\prime}\right)$, and we then do an $A \backslash\{a\}$ reverse step. If we did the $A \backslash\{a\}$ reverse step followed by the single $a$ reverse transition, then on the $\mathcal{D}$ side we leave open the possibility that $e^{\prime}$ causes the remaining events of $Y^{\prime} \cup\left\{e^{\prime}\right\} \stackrel{A \backslash\{a\}}{\longrightarrow} Y$.

In (2) we define $R^{\prime}$ in exactly the same way, and everything works much as before.

Combining:

Theorem 5.10. On stable configuration structures, $\approx_{r h s-i b}=\approx_{r s-s b}$.

Proof. Clearly any RS-SB is an RHS-IB. Any RHS-IB is an RS-SB, by Lemmas 5.8 and 5.9 .

\subsection{Reverse depth-preserving equivalences}

Recall that in the forward-only case, DB equivalence is strictly stronger than SB equivalence (Proposition 3.21). In this section we shall show that, by contrast, in the reverse case, the corresponding equivalences are equal (Theorem 5.24). The technical development will also be needed again when we later give an improvement of Bednarczyk's result that, in the absence of auto-concurrency, RI-IB equivalence has the same power as $\mathrm{H}-\mathrm{H}$ equivalence (Section 9).

We define reverse depth transitions and reverse equidepth transitions as follows:

Definition 5.11. Let $\mathcal{C}=(C, \ell)$ be a stable configuration structure and let $A \in \mathbb{N}^{\text {Act }}$, $k \in \mathbb{N}$. Then $X \stackrel{A, k}{\sim \mathcal{C}} X^{\prime}$ iff $X^{\prime} \stackrel{A, k}{\rightarrow} \mathcal{C} X$. Also $X \stackrel{A,=}{\leadsto \mathcal{C}} X^{\prime}$ iff $X^{\prime} \stackrel{A,=}{\rightarrow} \mathcal{C} X$.

This enables us to formulate equivalences $\approx_{r d-f b}$ and $\approx_{r d-h}$ in an analogous fashion to $\approx_{r s-f b}$ etc. We also have equidepth and homogeneous equidepth versions of the reverse step equivalences, denoted by $\approx_{r e s-f b}$ and $\approx_{r h e s-f b}$. We omit the definitions as the pattern is clear. 
We show that RS-SB equivalence can be characterised as RD-DB equivalence, and as RHES-IB equivalence.

Proposition 5.12. On stable configuration structures,

$1 \approx_{r d-i b} \subseteq \approx_{r s-i b} \subseteq \approx_{r h s-i b}$

$2 \approx_{r d-i b} \subseteq \approx_{r e s-i b} \subseteq \approx_{r h e s-i b}$.

Proof. These are reverse counterparts to inclusions already shown for the corresponding forward-only equivalences in Propositions 3.21 and 3.28, and are shown in much the same way.

We shall later show (Proposition 5.26) that all the equivalences mentioned in Proposition 5.12 are in fact equal to each other. First we need some further results.

Lemma 5.13. Let $\mathcal{C}, \mathcal{D}$ be stable configuration structures. Let $R$ be an RHES-IB between $\mathcal{C}$ and $\mathcal{D}$. If $R(X, Y)$ then $R(\min (X), \min (Y))$.

Proof. Similar to that of Lemma 5.4. Note that all events in $\min (X)$ have depth one, and any transition involving only minimal elements is an equidepth one.

Lemma 5.14. Let $\mathcal{C}, \mathcal{D}$ be stable configuration structures. Let $R$ be an RHES-IB between $\mathcal{C}$ and $\mathcal{D}$. Let $M \in C_{\mathcal{C}}$ be such that $\min (M)=M$. Similarly, let $N \in C_{\mathcal{D}}$ be such that $\min (N)=N$. Suppose also that $R(M, N)$. Define $R_{M, N}$ by

$$
R_{M, N}=\{(X \backslash M, Y \backslash N): R(X, Y), \min (X)=M, \min (Y)=N\}
$$

Then $R_{M, N}$ is an RHES-IB between $\mathcal{C}_{M}$ and $\mathcal{D}_{N}$.

Proof. Similar to that of Lemma 5.7, using Lemma 5.13 instead of Lemma 5.4.

Definition 5.15. Let $\mathcal{C}$ be a stable configuration structure. For $m, n \in \mathbb{N}(m \leq n)$ and $X \in C_{\mathcal{C}}$, let

$$
\begin{aligned}
& X_{\leq n} \stackrel{\text { df }}{=}\left\{e \in X: \operatorname{depth}_{X}(e) \leq n\right\} \\
& X_{\geq n} \stackrel{\text { df }}{=}\left\{e \in X: \operatorname{depth}_{X}(e) \geq n\right\} \\
& X_{[m, n]} \stackrel{\text { df }}{=}\left\{e \in X: m \leq \operatorname{depth}_{X}(e) \leq n\right\}
\end{aligned}
$$

Clearly $X_{\leq n}$ is a configuration, since it is a left-closed subset of a configuration. Also, $X_{\leq 1}=\min (X)$. For large enough $n, X_{\leq n}=X$.

Proposition 5.16. Suppose that $R$ is an RHES-IB between $\mathcal{C}$ and $\mathcal{D}$. If $R(X, Y)$ then for each $n \in \mathbb{N}$ we have $R\left(X_{\leq n}, Y_{\leq n}\right)$.

Proof. Suppose that $R(X, Y)$. We define configuration structures $\mathcal{C}_{n}, \mathcal{D}_{n}$ as follows:

$$
\begin{aligned}
& \mathcal{C}_{0} \stackrel{\text { df }}{=} \mathcal{C} \\
& \mathcal{C}_{n+1} \stackrel{\text { df }}{=}\left(\mathcal{C}_{n}\right)_{X_{[n+1, n+1]}}
\end{aligned}
$$

(see Definition 5.5), and similarly for $\mathcal{D}_{n}$. Note that $X_{[n+1, n+1]}=\min \left(X_{\geq n+1}\right)$. To ensure that $\mathcal{C}_{n}$ is well-defined, we show that $X_{\geq n+1}$ (and hence $X_{[n+1, n+1]}$ ) is a configuration of $\mathcal{C}_{n}$. This is easily done by induction on $n$ (omitted). Similarly for $\mathcal{D}_{n}$. 
We also define RHES-IBs $R_{n}$ as follows:

$$
\begin{aligned}
& R_{0} \stackrel{\text { df }}{=} R \\
& R_{n+1} \stackrel{\text { df }}{=}\left(R_{n}\right)_{X_{[n+1, n+1]}, Y_{[n+1, n+1]}}
\end{aligned}
$$

(see Lemma 5.14). To ensure that $R_{n+1}$ is well-defined (and an RHES-IB) we need $R_{n}\left(X_{[n+1, n+1]}, Y_{[n+1, n+1]}\right)$ to hold. We can prove $R_{n}\left(X_{\geq n+1}, Y_{\geq n+1}\right)$ by an easy induction, we omit. Using Lemma 5.13, we then deduce

$$
R_{n}\left(X_{[n+1, n+1]}, Y_{[n+1, n+1]}\right) .
$$

Now we show $R\left(X_{\leq n}, Y_{\leq n}\right)$. If $n=0$ this is just $R(\emptyset, \emptyset)$, which is true by definition. Suppose $n \geq 1$. We show by induction on $i$ that for $1 \leq i \leq n, R_{n-i}\left(X_{[n-i+1, n]}, Y_{[n-i+1, n]}\right)$. For $i=1$ this is just $R_{n-1}\left(X_{[n, n]}, Y_{[n, n]}\right)$, which we have already shown. Suppose that $R_{n-i}\left(X_{[n-i+1, n]}, Y_{[n-i+1, n]}\right)(i<n)$. Then

$$
R_{n-i-1}\left(X_{[n-i+1, n]} \cup X_{[n-i, n-i]}, Y_{[n-i+1, n]} \cup Y_{[n-i, n-i]}\right)
$$

i.e. $R_{n-(i+1)}\left(X_{[n-(i+1)+1, n]}, Y_{[n-(i+1)+1, n]}\right)$, as required.

Putting $i=n$, we get $R_{0}\left(X_{[1, n]}, Y_{[1, n]}\right)$, i.e. $R\left(X_{\leq n}, Y_{\leq n}\right)$.

Corollary 5.17. Suppose that $R$ is an RHES-IB between $\mathcal{C}$ and $\mathcal{D}$. If $R(X, Y)$ then for each $n \in \mathbb{N}$ we have $\ell\left(X_{[n, n]}\right)=\ell\left(Y_{[n, n]}\right)$.

Proof. By Proposition 5.16 and Lemma 5.3.

Proposition 5.18. Let $\mathcal{C}, \mathcal{D}$ be stable configuration structures. Suppose that $R$ is an RHES-IB between $\mathcal{C}$ and $\mathcal{D}$. If $X \stackrel{a, k}{\rightarrow} X^{\prime}, Y \stackrel{a, k^{\prime}}{\rightarrow} Y^{\prime}$, with $R(X, Y), R\left(X^{\prime}, Y^{\prime}\right)$, then $k=k^{\prime}$.

Proof. By Corollary 5.17, we know that $X, Y$ (resp. $X^{\prime}, Y^{\prime}$ ) have the same multisets of events at each level $n$. Hence the single events in $X^{\prime} \backslash X$ and $Y^{\prime} \backslash Y$ must have the same depth.

Lemma 5.19. Let $\mathcal{C}, \mathcal{D}$ be stable configuration structures, and let $R$ be an RHES-IB between $\mathcal{C}$ and $\mathcal{D}$. Then $R$ is an RD-DB.

Proof. Suppose that $R(X, Y)$ and $X \stackrel{a, k}{\rightarrow} X^{\prime}$. Since $R$ is an IB, there are $Y^{\prime}, k^{\prime}$ such that $Y \stackrel{a, k^{\prime}}{\rightarrow} Y^{\prime}$ and $R\left(X^{\prime}, Y^{\prime}\right)$. But then $k=k^{\prime}$ by Proposition 5.18. So $R$ is a DB. For the reverse transitions, suppose $R(X, Y)$ and $X \stackrel{a, k}{\cdots} \mathcal{C} X^{\prime}$. Then $X \stackrel{\{a\},=}{m \mathcal{C}} X^{\prime}$. Since $R$ is an RHES-IB, there is $Y^{\prime}$ such that $Y \stackrel{\{a\},=}{\sim} Y^{\prime}$ and $R\left(X^{\prime}, Y^{\prime}\right)$. Hence $Y \stackrel{a, k^{\prime}}{\rightsquigarrow \mathcal{D}} Y^{\prime}$ for some $k^{\prime}$. But then $k=k^{\prime}$, by a further use of Proposition 5.18. Thus $R$ is an RD-DB.

So if $R$ is an RHES-IB and $R\left(X, X^{\prime}\right)$ then $X$ and $X^{\prime}$ have similar structure, in that for each depth $n$, they have the same multisets of labelled events at that depth. We can say that $X$ and $X^{\prime}$ have similar amounts of concurrency (including auto-concurrency): $X$ and $X^{\prime}$ have the same depth, and the same "width" at each level $n$. Of course, this does not imply the stronger statement that $X$ and $X^{\prime}$ are isomorphic, since we are not claiming that $X$ and $X^{\prime}$ have the same causal relationships between the levels. 
Finally, we state versions of Proposition 5.16, Corollary 5.17, Proposition 5.18 and Lemma 5.19 for RHS-IBs instead of RHES-IBs.

Proposition 5.20. Suppose that $R$ is an RHS-IB between $\mathcal{C}$ and $\mathcal{D}$. If $R(X, Y)$ then for each $n \in \mathbb{N}$ we have $R\left(X_{\leq n}, Y_{\leq n}\right)$.

Proof. Similar to that of Proposition 5.16, using Lemmas 5.4 and 5.7 instead of Lemmas 5.13 and 5.14 .

Corollary 5.21. Suppose that $R$ is an RHS-IB between $\mathcal{C}$ and $\mathcal{D}$. If $R(X, Y)$ then for each $n \in \mathbb{N}$ we have $\ell\left(X_{[n, n]}\right)=\ell\left(Y_{[n, n]}\right)$.

Proof. By Proposition 5.20 and Lemma 5.3.

Proposition 5.22. Let $\mathcal{C}, \mathcal{D}$ be stable configuration structures. Suppose that $R$ is an RHS-IB between $\mathcal{C}$ and $\mathcal{D}$. If $X \stackrel{a, k}{\rightarrow} \mathcal{C} X^{\prime}, Y \stackrel{a, k^{\prime}}{\rightarrow} Y^{\prime}$, with $R(X, Y), R\left(X^{\prime}, Y^{\prime}\right)$, then $k=k^{\prime}$.

Proof. Similar to that of Proposition 5.18, using Corollary 5.21 instead of Corollary 5.17.

Lemma 5.23. Let $\mathcal{C}, \mathcal{D}$ be stable configuration structures, and let $R$ be an RHS-IB between $\mathcal{C}$ and $\mathcal{D}$. Then $R$ is an RD-DB.

Proof. Similar to that of Lemma 5.19, using Proposition 5.22 instead of Proposition 5.18.

Theorem 5.24. On stable configuration structures, $\approx_{r d-d b}=\approx_{r h e s-i b}=\approx_{r s-s b}$.

Proof. Let $R$ be an RD-DB between $\mathcal{C}$ and $\mathcal{D}$.

Then $R$ is an RHES-IB and an RHS-IB by Proposition 5.12. By Theorem 5.10, $R$ is therefore an RS-SB.

Now suppose $R$ is an RHES-IB. Then $R$ is an RD-DB by Lemma 5.19.

Finally, suppose $R$ is an RS-SB. Then $R$ is an RHS-IB, and so $R$ is an RD-DB by Lemma 5.23.

\subsection{The reverse step hierarchy}

We have seen in Section 5.1 that reverse steps are more powerful than forward steps: $\approx_{r s-i b} \subsetneq \approx_{r i-s b}$. We have also seen that reverse depth observations are equivalent to reverse steps (Theorem 5.24).

As a consequence, there is quite a collapse in the hierarchy of equivalences in the "reverse step family", compared to the reverse single-event equivalences.

Proposition 5.25. Let $\mathcal{C}, \mathcal{D}$ be stable configuration structures. The following are equivalent:

$1 \mathcal{C} \approx_{r s-i b} \mathcal{D}$

$2 \mathcal{C} \approx_{r s-d b} \mathcal{D}$

$3 \mathcal{C} \approx_{r s-s b} \mathcal{D}$

$4 \mathcal{C} \approx_{r s-h s b} \mathcal{D}$; 
$5 \mathcal{C} \approx_{r s-e s b} \mathcal{D}$

$6 \mathcal{C} \approx_{r s-h e s b} \mathcal{D}$.

Proof. The implications $(2) \Rightarrow(3) \Rightarrow(4) \Rightarrow(1)$ and $(2) \Rightarrow(5) \Rightarrow(6) \Rightarrow(1)$ hold by the corresponding results for the forward-only equivalences as shown in Figure 7. Hence it is enough to show $(1) \Rightarrow(2)$.

Suppose $R$ is an RS-IB. Then it is an RHS-IB. So it is an RD-DB by Lemma 5.23. Combining, $R$ is an RS-DB, as required.

Proposition 5.26. Let $\mathcal{C}, \mathcal{D}$ be stable configuration structures. The following are equivalent:

$1 \quad R$ is an RS-IB;

$2 \quad R$ is an RHS-IB;

$3 \quad R$ is an RD-IB;

$4 \quad R$ is an RES-IB;

$5 \quad R$ is an RHES-IB.

Proof. $(1) \Rightarrow(2)$ is immediate from the definition.

$(2) \Rightarrow(3)$ holds by Lemma 5.23 .

$(3) \Rightarrow(4)$ holds by (the proof of) Proposition 5.12.

$(4) \Rightarrow(5)$ is immediate from the definition.

$(5) \Rightarrow(3)$ holds by Lemma 5.19 .

$(3) \Rightarrow(1)$ holds by (the proof of) Proposition 5.12.

Corollary 5.27. Let $\approx_{f b}$ be any of the forward-only equivalences in Figure 7 . Then on stable configuration structures we have $\approx_{r s-f b}=\approx_{r h s-f b}=\approx_{r d-f b}=\approx_{r e s-f b}=\approx_{r h e s-f b}$.

Proof. Immediate from Proposition 5.26.

Proposition 5.28. Let $\mathcal{C}, \mathcal{D}$ be stable configuration structures. Let $R$ be an RI-WH bisimulation between $\mathcal{C}$ and $\mathcal{D}$. Then $R$ is an RD-WH bisimulation.

Proof. Suppose that $R(X, Y)$, and $X \stackrel{a, k}{\sim \rightarrow} \mathcal{C} X^{\prime}$. Then $X \stackrel{a}{\sim} \mathfrak{C} X^{\prime}$, and so there is $Y^{\prime}$ such that $Y \stackrel{a}{\rightsquigarrow} \mathcal{C} Y^{\prime}$ with $R\left(X^{\prime}, Y^{\prime}\right)$. Since $R$ is a WH bisimulation, $X \cong Y$ and $X^{\prime} \cong Y^{\prime}$. Let $X \backslash X^{\prime}=\{e\}$ and $Y \backslash Y^{\prime}=\left\{e^{\prime}\right\}$. Then $\operatorname{depth}_{X}(e)=\operatorname{depth}_{Y}\left(e^{\prime}\right)$, since depth is preserved by isomorphism. Hence $Y \stackrel{a, k}{\rightarrow} \mathcal{C} Y^{\prime}$. Therefore $R$ is an RD-WH as required.

Corollary 5.29. On stable configuration structures, we have $\approx_{r s-w h}=\approx_{r i-w h}, \approx_{r s-w h p b}=$ $\approx_{r i-w h p b}$ and $\approx_{r s-h}=\approx_{r i-h}$.

Proof. Immediate from Proposition 5.28 and Corollary 5.27.

Figure 18 shows the reverse single-event or step equivalences arranged in a single ordering. Recall that the open arrows represent inclusions which are not known to be proper, and the solid arrows represent proper inclusions. Equivalences $\approx_{r s-s b}$ and $\approx_{r s-d b}$ are the same as $\approx_{r s-i b}$ by Proposition 5.25, and the remaining equations hold by Corollary 5.29. The various inclusions follow from the results in this section. These have all been established in Section 4, with two exceptions: 


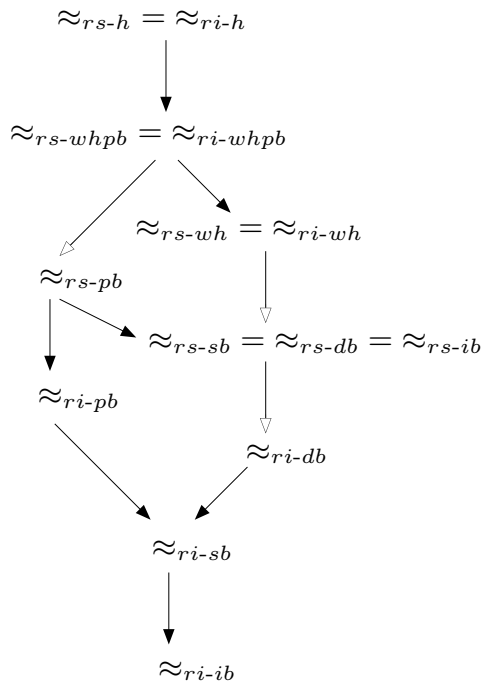

Fig. 18. Equivalences with reverse single-event or step transitions.

- For $\approx_{r s-p b} \subsetneq \approx_{r i-p b}$ we use Example 4.6 , where the two structures are RI-PB equivalent, but not DB equivalent. Hence they are not RI-DB equivalent. But from Figure 18 we see that they must therefore be not RS-PB equivalent.

- For $\approx_{r s-p b} \subsetneq \approx_{r s-s b}$ we use Example 4.7 , where the two structures are RI-WH equivalent, but not PB equivalent. They are therefore RS-WH, and so RS-SB, equivalent, but not RS-PB equivalent.

We conclude by noting that extending the allowed observations to include reverse step transitions increases of the distinguishing power of the coarser equivalences in Figure 8, namely $\approx_{r s-i b} \subsetneq \approx_{r i-i b}, \approx_{r s-s b} \subsetneq \approx_{r i-s b}$ and $\approx_{r s-p b} \subsetneq \approx_{r i-p b}$ in Figure 18. It, however, has has no effect on the three finer history-preserving-like equivalences $\approx_{r i-w h}, \approx_{r i-w h p b}$ and $\approx_{r i-h}$ as we have $\approx_{r s-w h}=\approx_{r i-w h}, \approx_{r s-w h p b}=\approx_{r i-w h p b}$ and $\approx_{r s-w h}=\approx_{r i-w h}$.

\section{Reverse pomset transitions}

The reverse pomset transition relation, with a wavy arrow, is defined in a corresponding way to the single event reverse transition, namely by simply inverting the standard forward pomset version of Definition 3.6.

Definition 6.1. Let $\mathcal{C}=(C, \ell)$ be a stable configuration structure and let $u$ be a pomset as in Definition 3.5. Then $X \stackrel{u}{\sim} \mathcal{C} X^{\prime}$ iff $X^{\prime} \stackrel{u}{\rightarrow} \mathcal{C} X$.

Much as for single reverse event transitions, we can now define reverse pomset versions $\approx_{r p-f b}$ and $\approx_{r p-h}$ of all the equivalences defined in Section 3. We omit the definitions, which are similar to Definitions 4.2 and 4.3 . We can define equivalences $\approx_{r h s-f b}$ in a corresponding way. 


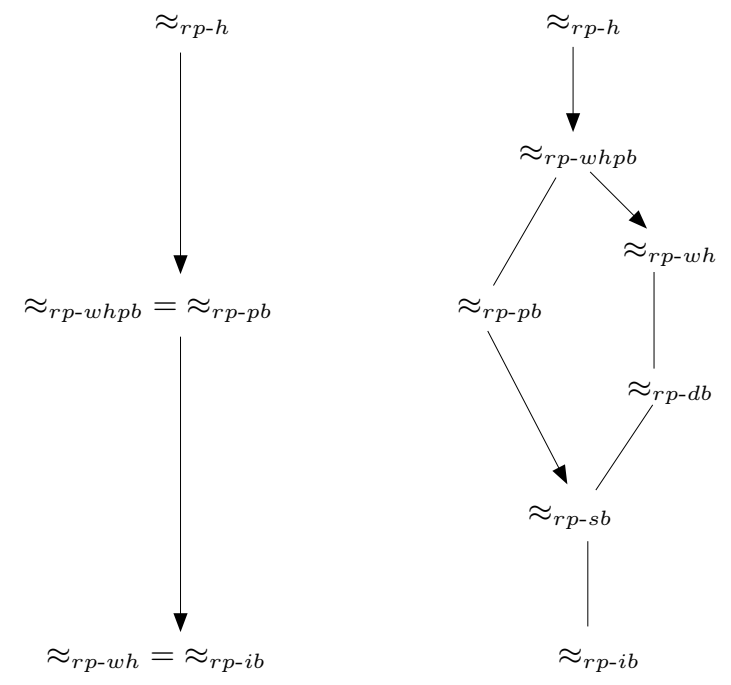

Fig. 19. Equivalences with reverse pomset transitions.

We observe that any of these bisimulations gives us an order isomorphism. Assume

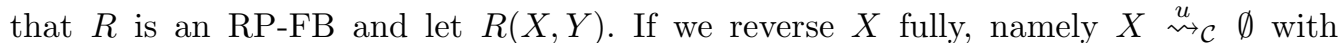
$u=\left[\left(X,<_{X}, \ell_{\mathcal{C}}\lceil X)\right] \cong\right.$, then this must be matched by $Y \stackrel{u}{\sim} \mathcal{D} \emptyset$ by the definition of $R$. Thus, $u$ is the pomset associated to $Y$ and $X \cong Y$. As a consequence, an RP-IB is also an RP-WH and an RP-PB is also an RP-WHPB:

Theorem 6.2. On stable configuration structures, $\approx_{r p-i b}=\approx_{r p-s b}=\approx_{r p-d b}=\approx_{r p-w h}$. And, $\approx_{r p-p b}=\approx_{r p-w h p b}$.

Example 4.7 holds for $\approx_{r p-s b}$, but not for $\approx_{r p-p b}$. And it holds for $\approx_{r p-w h}$, but not for $\approx_{r p-w h p b}$. Finally, Example 4.8 shows that $\approx_{r p-h}$ is strictly contained in $\approx_{r p-w h p b}$.

Figure 19 shows the full hierarchy of reverse pomset equivalences on the right. Plain lines indicate coincidence of relations. We notice that there are only three distinct relations, and so when we take conceptually simplest relations as the representatives of the sets of coinciding bisimulations, we obtain the left hierarchy in Figure 19. It is an open question whether the reverse pomset equivalences strictly imply the corresponding reverse step equivalences (e.g. whether $\approx_{r p-h} \subsetneq \approx_{r s-h}$ ).

\section{Hereditary equivalences}

The reverse versions of history preserving bisimulation, weak history bisimulation and weak history pomset bisimulation are defined by additionally insisting that the related configurations have also matching reverse behaviour (reverse single events, reverse steps or reverse pomsets). The hereditary property states that if there is an order isomorphism between two configurations, this isomorphism is preserved under reverse single event transitions. Therefore, correspondingly as in the definition of history-preserving bisim- 
ulation (Definition 3.10), it is useful to name the isomorphism explicitly when defining WH and WHPB. We restate these definitions for clarity.

Definition 7.1. Let $\mathcal{C}, \mathcal{D}$ be stable configuration structures. A relation $R \subseteq C_{\mathcal{C}} \times C_{\mathcal{D}} \times$ $\mathcal{P}\left(E_{\mathcal{C}} \times E_{\mathcal{D}}\right)$ is a weak history-preserving $(W H)$ bisimulation between $\mathcal{C}$ and $\mathcal{D}$ if $(\emptyset, \emptyset, \emptyset) \in$ $R$ and if $(X, Y, f) \in R$ and $a \in$ Act

$-f$ is an isomorphism between $\left(X,<_{X}, \ell_{\mathcal{C}}\lceil X)\right.$ and $\left(Y,<_{Y}, \ell_{\mathcal{D}} \backslash Y\right)$;

- if $X \stackrel{a}{\rightarrow} \mathcal{C} X^{\prime}$ then $\exists Y^{\prime}, f^{\prime} . Y \stackrel{a}{\rightarrow} \mathcal{D} Y^{\prime}$ and $\left(X^{\prime}, Y^{\prime}, f^{\prime}\right) \in R$;

- if $Y \stackrel{a}{\rightarrow} \mathcal{D} Y^{\prime}$ then $\exists X^{\prime}, f^{\prime} . X \stackrel{a}{\rightarrow} \mathcal{C} X^{\prime}$ and $\left(X^{\prime}, Y^{\prime}, f^{\prime}\right) \in R$.

We say that $\mathcal{C}$ and $\mathcal{D}$ are WH equivalent $\left(\mathcal{C} \approx_{w h} \mathcal{D}\right)$ iff there is a WH bisimulation between $\mathcal{C}$ and $\mathcal{D}$.

Definition 7.2. Let $\mathcal{C}, \mathcal{D}$ be stable configuration structures. A relation $R \subseteq C_{\mathcal{C}} \times C_{\mathcal{D}} \times$ $\mathcal{P}\left(E_{\mathcal{C}} \times E_{\mathcal{D}}\right)$ is a weak history-preserving pomset bisimulation (WHPB) between $\mathcal{C}$ and $\mathcal{D}$ if $(\emptyset, \emptyset, \emptyset) \in R$ and if $(X, Y, f) \in R$ and $u$ is a pomset over Act then

$-f$ is an isomorphism between $\left(X,<_{X}, \ell_{\mathcal{C}}\lceil X)\right.$ and $\left(Y,<_{Y}, \ell_{\mathcal{D}} \mid Y\right)$;

- if $X \stackrel{u}{\rightarrow} \mathcal{C} X^{\prime}$ then $\exists Y^{\prime}, f^{\prime} . Y \stackrel{u}{\rightarrow}_{\mathcal{D}} Y^{\prime}$ and $\left(X^{\prime}, Y^{\prime}, f^{\prime}\right) \in R$;

— if $Y \stackrel{u}{\rightarrow} \mathcal{D} Y^{\prime}$ then $\exists X^{\prime}, f^{\prime} . X \stackrel{u}{\rightarrow} \mathcal{C} X^{\prime}$ and $\left(X^{\prime}, Y^{\prime}, f^{\prime}\right) \in R$.

We say that $\mathcal{C}$ and $\mathcal{D}$ are WHPB equivalent $\left(\mathcal{C} \approx_{w h p b} \mathcal{D}\right)$ iff there is a WHPB between $\mathcal{C}$ and $\mathcal{D}$.

We can now define hereditary versions of the three forward equivalences that use history isomorphisms. Let FH stand for any of WH, WHPB and $\mathrm{H}$, and let $\approx_{f h}$ be the associated equivalence.

Definition 7.3. Let $\mathcal{C}=(C, \ell)$ be a stable configuration structure and let $a \in$ Act. Then $R \subseteq C_{\mathcal{C}} \times C_{\mathcal{D}} \times \mathcal{P}\left(E_{\mathcal{C}} \times E_{\mathcal{D}}\right)$ is an H-FH iff $R$ is an FH and if $R(X, Y, f)$ then for any $a \in$ Act,

— if $X \stackrel{a}{\rightsquigarrow \mathcal{C}} X^{\prime}$ then $\exists Y^{\prime}, f^{\prime} . Y \stackrel{a}{\rightsquigarrow \mathcal{D}} Y^{\prime},\left(X^{\prime}, Y^{\prime}, f^{\prime}\right) \in R$ and $f\left\lceil X^{\prime}=f^{\prime}\right.$;

— if $Y \stackrel{a}{\rightsquigarrow} \mathcal{D} Y^{\prime}$ then $\exists X^{\prime}, f^{\prime} . X \stackrel{a}{\rightsquigarrow} \mathcal{C} X^{\prime},\left(X^{\prime}, Y^{\prime}, f^{\prime}\right) \in R$ and $f\left\lceil X^{\prime}=f^{\prime}\right.$.

We say that $\mathcal{C}$ and $\mathcal{D}$ are $\mathrm{H}-\mathrm{FH}$ equivalent $\left(\mathcal{C} \approx_{h-f h} \mathcal{D}\right)$ iff there is an H-FH bisimulation between $\mathcal{C}$ and $\mathcal{D}$.

The hereditary property is due to Bednarczyk (1991). It is shown in (van Glabbeek and Goltz 2001) that the property is invariant under replacement of the single event transitions by step or pomset transitions when used in the definition of $\mathrm{H}-\mathrm{H}$ bisimulation.

It is clear by the definitions of H-H, H-WHPB and H-WH that $\approx_{h-h} \subseteq \approx_{h-w h p b} \subseteq$ $\approx_{h-w h}$. Example 4.7 shows that $\approx_{h-w h p b}$ is strictly contained in $\approx_{h-w h}$ and Example 4.8 shows that $\approx_{h-h}$ is strictly contained in $\approx_{h-w h p b}$.

Figure 20 gives the full hierarchy of hereditary equivalences and reverse pomset equivalences (on the right). Open question remains if the hereditary equivalences are strictly finer than the corresponding reverse pomset equivalences. 


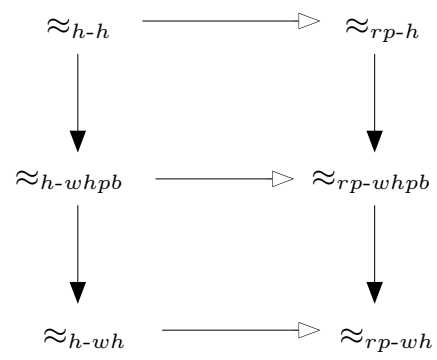

Fig. 20. Hereditary equivalences and equivalences with reverse pomset transitions.

\section{Refinement}

van Glabbeek and Goltz (2001) investigated refinement of actions, and showed that the finest two bisimulation equivalences they investigated, namely $\approx_{h}$ and $\approx_{h-h}$, are preserved under refinement, whereas the other bisimulation equivalences they considered, namely $\approx_{i b}, \approx_{s b}, \approx_{p b}, \approx_{w h}$ and $\approx_{w h p b}$ are not. We strengthen their negative results by showing that any equivalence lying between $\approx_{h-w h p b}$ and $\approx_{i b}$ is not preserved under refinement. We refer the reader to (van Glabbeek and Goltz 2001, Definition 5.4) for the definition of a refinement function $r e f$ and the refinement $\operatorname{ref}(\mathcal{C})$ of a stable configuration structure $\mathcal{C}$.

Proposition 8.1. Let $\approx_{*}$ be such that $\approx_{h-w h p b} \subseteq \approx_{*} \subseteq \approx_{i b}$. Then $\approx_{*}$ is not preserved by refinement.

Proof. Consider the structures $\mathcal{E}, \mathcal{F}$ of Example 4.8. We have $\mathcal{E} \approx_{h \text {-whpb }} \mathcal{F}$. Hence $\mathcal{E} \approx_{*} \mathcal{F}$. If we refine $a$ to the structure $a^{\prime} \rightarrow a^{\prime \prime}$ (i.e. $\operatorname{ref}(a)=a^{\prime} \rightarrow a^{\prime \prime}$ ) then in $\operatorname{ref}(\mathcal{E})$, any sequence of transitions $\stackrel{a^{\prime}}{\rightarrow} \stackrel{a^{\prime \prime}}{\rightarrow} \stackrel{a^{\prime}}{\rightarrow}$ leaves $\stackrel{b}{\rightarrow}$ possible. However in $\operatorname{ref}(\mathcal{F})$ if we perform $\stackrel{a_{3}^{\prime}}{\rightarrow} \stackrel{a_{3}^{\prime \prime}}{\rightarrow} \stackrel{a_{2}^{\prime}}{\rightarrow}$ then $\stackrel{b}{\rightarrow}$ is impossible; see Figure 21 . Thus $\operatorname{ref}(\mathcal{E}) \not_{i b} \operatorname{ref}(\mathcal{F})$, and so $\operatorname{ref}(\mathcal{E}) \not_{*}$ $\operatorname{ref}(\mathcal{F})$.

We leave as an open question whether the equivalences lying between $\approx_{h}$ and $\approx_{h-h}$, namely $\approx_{r i-h}$ and $\approx_{r p-h}$, are preserved by refinement. As we have seen, the inclusions $\approx_{h-h} \subseteq \approx_{r p-h} \subseteq \approx_{r i-h}$ may or may not be strict.

\section{Excluding equidepth auto-concurrency}

In this section we extend a result of Bednarczyk which states that RI-IB equivalence is as strong as H-H equivalence in the absence of auto-concurrency. We make use of results from Section 5.2 on depth-respecting bisimulations, and show that Bednarczyk's result still holds under the weaker assumption of no equidepth auto-concurrency. We conclude the section by showing the complete (and considerably simplified) hierarchy of equivalences under this assumption. 

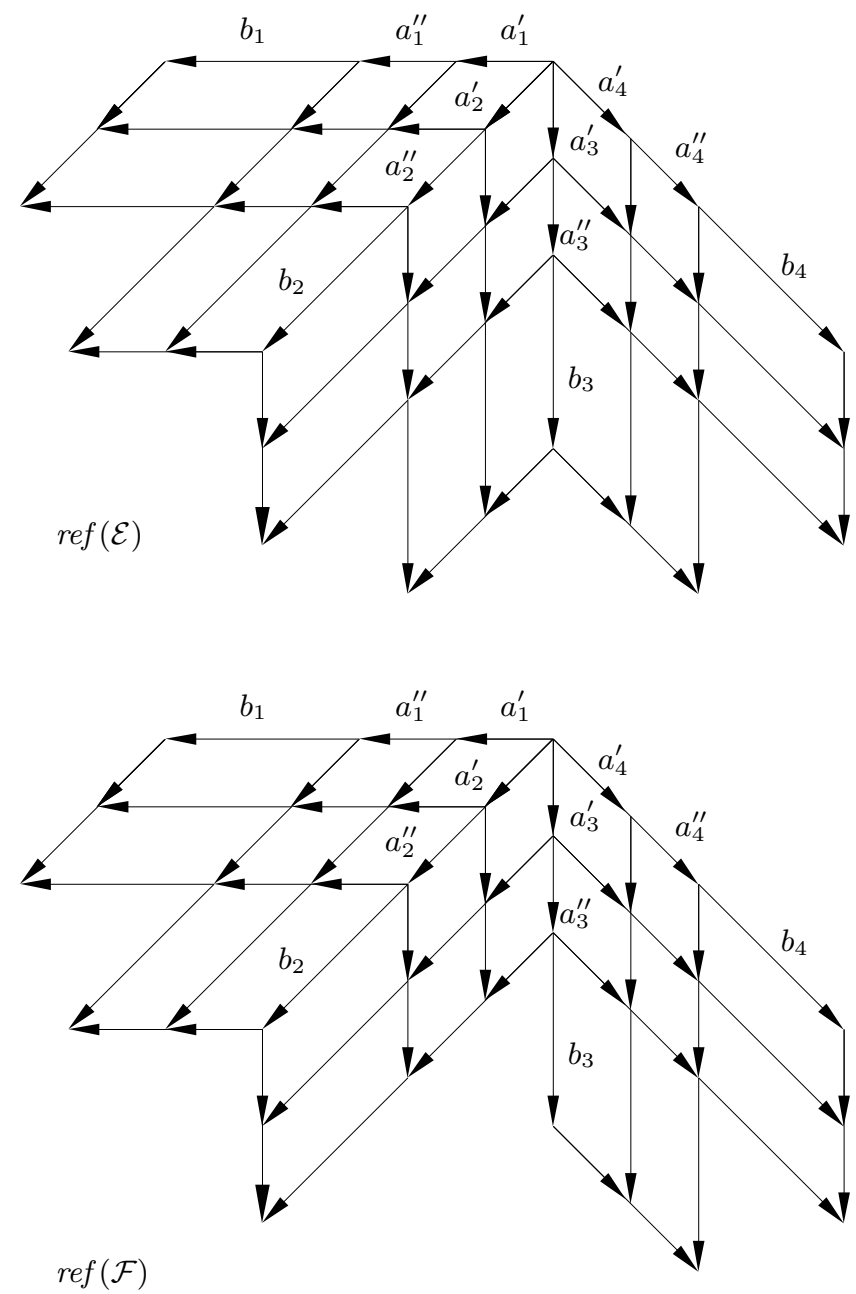

Fig. 21. Transition diagrams for $\operatorname{ref}(\mathcal{E})$ and $\operatorname{ref}(\mathcal{F})$.

Definition 9.1. We say that $\mathcal{C}$ be a stable configuration structure is without autoconcurrency if for any $X \in C_{\mathcal{C}}$ and any $d, e \in X$, if $d \operatorname{co}_{X} e$ and $\ell(d)=\ell(e)$ then $d=e$.

Bednarczyk (1991) showed the following:

Theorem 9.2. For prime event structures, in the absence of auto-concurrency, $\approx_{r i-i b}=$ $\approx_{h-h}$.

Before extending Theorem 9.2 in Theorem 9.7 below, we need a definition. We say that a configuration structure is without equidepth auto-concurrency, if for any pair of distinct concurrent events, they cannot have both the same label and the same depth. This is less restrictive than "without auto-concurrency", in that we allow auto-concurrent 
events, providing they are at different depths. For example, the configuration structure $a \mid(b . a)$ exhibits auto-concurrency between the two events labelled $a$, but not equidepth auto-concurrency, since the two $a$ events are at depths one and two respectively.

Definition 9.3. We say that a stable configuration structure $\mathcal{C}$ is without equidepth auto-concurrency if for any $X \in C_{\mathcal{C}}$ and any $d, e \in X$, if $d \operatorname{co}_{X} e$ with both $\ell(d)=\ell(e)$ and $\operatorname{depth}_{X}(d)=\operatorname{depth}_{X}(e)$, then $d=e$.

Notice that without equidepth auto-concurrency, an RI-IB is an RHES-IB, since all reverse homogeneous equidepth steps must have just a single event: $X \stackrel{A,}{\sim} \stackrel{\overline{\mathcal{C}}}{X^{\prime}}$ with $A$ homogeneous implies that $|A|=1$. Therefore we immediately have the following versions of Proposition 5.16, Corollary 5.17 and Proposition 5.18, where $X_{\leq n}$ and $X_{[n, n]}$ are as in Definition 5.15:

Proposition 9.4. Let $\mathcal{C}, \mathcal{D}$ be stable configuration structures without equidepth autoconcurrency. Suppose that $R$ is an RI-IB between $\mathcal{C}$ and $\mathcal{D}$. If $R(X, Y)$ then for each $n \in \mathbb{N}$ we have $R\left(X_{\leq n}, Y_{\leq n}\right)$.

Corollary 9.5. Let $\mathcal{C}, \mathcal{D}$ be stable configuration structures without equidepth autoconcurrency. Suppose that $R$ is an RI-IB between $\mathcal{C}$ and $\mathcal{D}$. If $R(X, Y)$ then for each $n \in \mathbb{N}$ we have $\ell\left(X_{[n, n]}\right)=\ell\left(Y_{[n, n]}\right)$.

Note that the no equidepth auto-concurrency condition means that $\ell\left(X_{[n, n]}\right), \ell\left(Y_{[n, n]}\right)$ are sets rather than multisets, since events at the same depth must be concurrent.

Proposition 9.6. Let $\mathcal{C}, \mathcal{D}$ be stable configuration structures without equidepth autoconcurrency. Suppose that $R$ is an RI-IB between $\mathcal{C}$ and $\mathcal{D}$. If $X \stackrel{a, k}{\rightarrow} \mathcal{C} X^{\prime}, Y \stackrel{a, k^{\prime}}{\rightarrow} Y^{\prime}$, with $R(X, Y), R\left(X^{\prime}, Y^{\prime}\right)$, then $k=k^{\prime}$.

Proposition 9.6 would not necessarily hold in the presence of equidepth auto-concurrency. For example, we have $a \mid a \approx_{r i-i b} a . a$, but the two $a$ events are both at depth one in the case of $a \mid a$, whereas they are at depths one and two respectively in the case of a.a.

Theorem 9.7. In the absence of equidepth auto-concurrency, $\approx_{r i-i b}=\approx_{h-h}$.

Proof. The proof is rather different from that of Theorem 9.2, and comes out quite rapidly using Corollary 9.5 and Proposition 9.6. Let $\mathcal{C}, \mathcal{D}$ be stable configuration structures without equidepth auto-concurrency. Suppose that $R$ is an RI-IB between $\mathcal{C}$ and $\mathcal{D}$.

Suppose that $R(X, Y)$. Define $f_{X, Y}: X \rightarrow Y$ by $f_{X, Y}(d)=e$ where $e$ is the unique $e \in Y$ such that $\operatorname{depth}_{Y}(e)=\operatorname{depth}_{X}(d)$ and $\ell(e)=\ell(d)$. Then $f_{X, Y}$ is well-defined and is a bijection by Corollary 9.5. Clearly $f$ preserves labels and depth.

We next show $f_{X, Y}$ is order-preserving. Note that events in $X$ and $Y$ are determined uniquely by their depth and label. Suppose $d<_{X} d^{\prime}$, with $\ell(d)=a, \ell\left(d^{\prime}\right)=a^{\prime}$, $\operatorname{depth}_{X}(d)=k, \operatorname{depth}_{X}\left(d^{\prime}\right)=k^{\prime}$. Suppose for a contradiction that $f_{X, Y}(d) \nless_{Y} f_{X, Y}\left(d^{\prime}\right)$. Then there is $Y^{\prime \prime} \in C_{\mathcal{D}}$ such that $f_{X, Y}\left(d^{\prime}\right) \in Y^{\prime \prime} \subsetneq Y$ and $f_{X, Y}(d) \notin Y^{\prime \prime}$. So there is a series of reverse transitions starting from $Y$ in which we reverse $f_{X, Y}(d)$ but not 
$f_{X, Y}\left(d^{\prime}\right)$, i.e. $Y \stackrel{a_{1}, k_{1}}{\rightsquigarrow \mathcal{D}} Y_{1} \ldots \stackrel{a_{n}, k_{n}}{\rightsquigarrow \mathcal{D}} Y_{n} \stackrel{a, k}{\stackrel{a}{\rightsquigarrow \mathcal{D}}} Y^{\prime} \supseteq Y^{\prime \prime}$, with $\ell\left(a_{i}\right) \neq a^{\prime}$ or $\operatorname{depth}_{Y}\left(a_{i}\right) \neq k^{\prime}$ for $i=1, \ldots, n$.

Since $R$ is an RI-IB, there is a corresponding series of reverse transitions starting from $X$. The corresponding events must have the same depth, by Proposition 9.6. So $X \stackrel{a_{1}, k_{1}}{\sim} X_{1} \ldots \stackrel{a_{n}, k_{n}}{\sim} X_{n} \stackrel{a, k}{\sim} \underset{\sim}{*} X^{\prime}$, with $R\left(X_{i}, Y_{i}\right)$ for $i=1, \ldots, n$ and $R\left(X^{\prime}, Y^{\prime}\right)$.

But then the last transition $X_{n} \stackrel{a, k}{\rightarrow \mathcal{C}} X^{\prime}$ must have $d$ as its underlying event, and none of the previous $n$ transitions can have $d^{\prime}$ as underlying event, since either the depth or label does not match. This means that $d \nless_{X} d^{\prime}$, which is a contradiction.

Symmetrically we can show that $e<_{Y} e^{\prime}$ implies $f_{X, Y}^{-1}(e)<_{X} f_{X, Y}^{-1}(e)$ (the definition of $f_{X, Y}^{-1}$ is just the same as that of $f_{X, Y}$ with $X$ and $Y$ swapped). Hence $f_{X, Y}$ is orderpreserving.

Claim 1. If $X \stackrel{a}{\rightarrow} \mathcal{C} X^{\prime}, Y \stackrel{a}{\rightarrow} \mathcal{D} Y^{\prime}$ and $R(X, Y), R\left(X^{\prime}, Y^{\prime}\right)$, then $f_{X^{\prime}, Y^{\prime}} \uparrow X=f_{X, Y}$.

Proof of Claim: Let $X^{\prime} \backslash X=\{d\}, Y^{\prime} \backslash Y=\{e\}$. We first show $f_{X^{\prime}, Y^{\prime}}(d)=e$. By Proposition 9.6 there is $k$ such that $X \stackrel{a, k}{\rightarrow} \mathcal{C} X^{\prime}$ and $Y \stackrel{a, k}{\rightarrow} \mathcal{D} Y^{\prime}$. $\operatorname{So~depth}_{Y^{\prime}}(e)=\operatorname{depth}_{X^{\prime}}(d)$ and $\ell(e)=\ell(d)$. Hence $f_{X^{\prime}, Y^{\prime}}(d)=e$ by definition of $f_{X^{\prime}, Y^{\prime}}$. Take any $d^{\prime} \in X$. Then $f_{X^{\prime}, Y^{\prime}}\left(d^{\prime}\right)=e^{\prime}$ where $e^{\prime}$ is the unique $e^{\prime} \in Y^{\prime}$ such that $\operatorname{depth}_{Y^{\prime}}\left(e^{\prime}\right)=\operatorname{depth}_{X^{\prime}}\left(d^{\prime}\right)$ and $\ell\left(e^{\prime}\right)=\ell\left(d^{\prime}\right)$. Since $f_{X^{\prime}, Y^{\prime}}(d)=e$, we must have $e^{\prime} \in Y$. Hence $f_{X^{\prime}, Y^{\prime}}\left(d^{\prime}\right)=f_{X, Y}\left(d^{\prime}\right)$, and the Claim is shown.

Now define $R^{\prime}(X, Y, f)$ iff $R(X, Y)$ and $f=f_{X, Y}$ (any $X, Y$ ). We claim that $R^{\prime}$ is an H-H bisimulation between $\mathcal{C}$ and $\mathcal{D}$. We have shown that $f_{X, Y}$ is an isomorphism between $\left(X,<_{X}, \ell_{\mathcal{C}}\lceil X)\right.$ and $\left(Y,<_{Y}, \ell_{\mathcal{D}}\lceil Y)\right.$. Clearly $R^{\prime}\left(\emptyset, \emptyset, f_{\emptyset, \emptyset}\right)$. Assume $R^{\prime}(X, Y, f)$.

Suppose $X \stackrel{a}{\rightarrow} \mathcal{C} X^{\prime}$. Then there is $Y^{\prime}$ such that $Y \stackrel{a}{\rightarrow} \mathcal{D} Y^{\prime}$ and $R\left(X^{\prime}, Y^{\prime}\right)$. So we obtain $R^{\prime}\left(X^{\prime}, Y^{\prime}, f_{X^{\prime}, Y^{\prime}}\right)$. Also $f_{X^{\prime}, Y^{\prime}} \uparrow X=f_{X, Y}$ by the Claim above.

The remaining cases where we suppose $Y \stackrel{a}{\rightarrow} \mathcal{D} Y^{\prime}$ and $X \stackrel{a}{{ }^{\prime}} \mathcal{C} X^{\prime}$ are handled similarly, again using the Claim.

We have strengthened Theorem 9.2 in two ways: we use stable configuration structures rather than prime event structures, and we weaken the assumption of no autoconcurrency to no equidepth auto-concurrency.

Figure 22 shows the complete hierarchy of equivalences under the condition of no equidepth auto-concurrency. Since all the reverse equivalences are intermediate between $\approx_{h-h}$ and $\approx_{r i-i b}$, they all collapse to $\approx_{h-h}=\approx_{r i-i b}$ by Theorem 9.7 .

van Glabbeek and Goltz (2001) showed that in the absence of auto-concurrency, $\approx_{h}=$ $\approx_{w h}$ (Theorem 9.2). It is not hard to check that their proof still works in the absence of equidepth auto-concurrency. Of course, $\approx_{w h p b}$ is then also the same as $\approx_{h}$. Finally, as already observed earlier $\approx_{\text {hesb }}=\approx_{i b}$.

The inclusions in Figure 22 follow from those in Figure 7. All the arrows represent strict inclusions, and no other inclusions hold. We now provide examples to show that this is the case. We have to be careful not to use examples with equidepth auto-concurrency.

Example 9.8. Let us modify processes $\mathcal{E}$ and $\mathcal{F}$ of Example 3.30 by adding a new event labelled with $c$ in parallel to form new processes $\mathcal{E}^{\prime \prime}$ and $\mathcal{F}^{\prime \prime}$. See Figure 23. Then $\mathcal{E}^{\prime \prime} \approx_{p b} \mathcal{F}^{\prime \prime}$ but $\mathcal{E}^{\prime \prime} \nsim_{e s b} \mathcal{F}^{\prime \prime}$. 


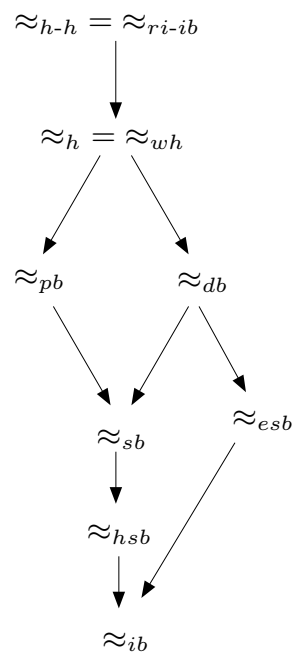

Fig. 22. The hierarchy with no equidepth auto-concurrency.

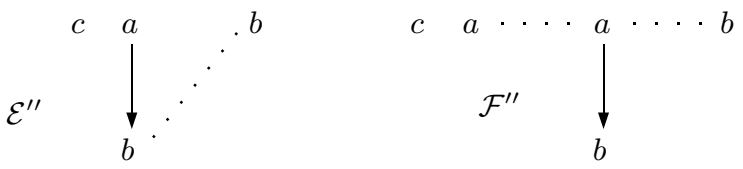

Fig. 23. Example 9.8.

Example 9.9. $a \mid(b . b)=(a \mid(b . b))+b .(a \mid b)$ (where $a \neq b)$ holds for $\approx_{s b}$ but not for $\approx_{p b}$ or $\approx_{e s b}$.

We can complete the task of showing that all arrows represent strict inclusions, and that no other inclusions hold, using existing examples, as follows:

- Example 3.27 holds for $\approx_{e s b}$ but not for $\approx_{h s b}$;

- Example 3.26 holds for $\approx_{h s b}$ but not for $\approx_{s b}$ or $\approx_{e s b}$;

- Example 3.22 holds for $\approx_{d b}$ but not for $\approx_{p b}$;

- Example 4.9 holds for $\approx_{h}$ but not for $\approx_{h-h}=\approx_{r i-i b}$.

\section{Concluding remarks}

We have investigated several forms of observations of reversible behaviour and have defined a wide range of reverse bisimulations by combining both forward and reverse observations. We have considered reverse single-event transitions, reverse steps, reverse depth-preserving transitions, reverse pomsets and the hereditary property of reverse behaviour.

Figure 24 shows relationships between the main forward-only equivalences and the range of reverse versions of these equivalences. Solid-headed arrows represent proper inclusions, open-headed arrows represent an inclusion not known to be proper and plain 


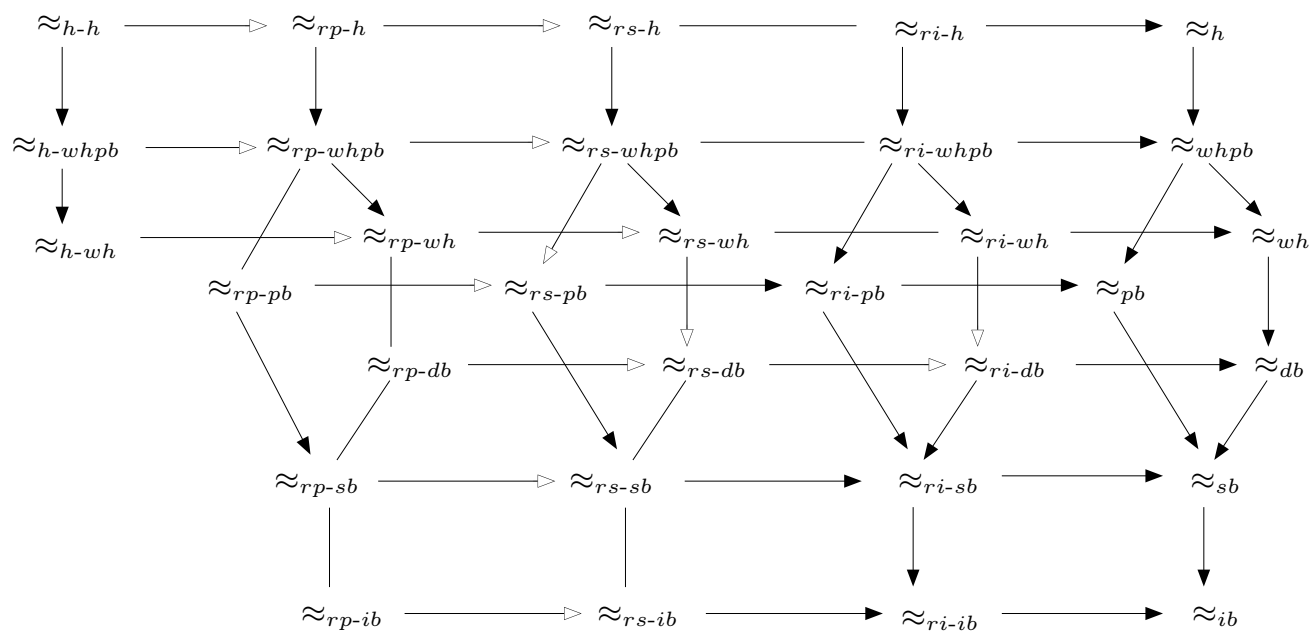

Fig. 24. The hierarchy of forward and reverse bisimulations.

lines represent coincidence of equivalences. We recall that the absorption law (Example 4.9) separates the forward-only hierarchy from all the reverse bisimulations, and Example 4.8 separates the top row equivalences, namely those between $\approx_{h}$ and $\approx_{h-h}$, from all the remaining equivalences. Example 4.7 distinguishes $\approx_{w h p b}$ from $\approx_{w h}$ and it distinguishes all the $r i-, r s-, r p$ - and $h$ - versions of the two equivalences. Moreover, it separates $\approx_{p b}$ and $\approx_{s b}$ and all the $r i-, r s-, r p$ - and $h$ - versions of these bisimulations. There are still open questions about whether the equivalences related by open-headed arrows in Figure 24 are distinct. We conjecture that they are indeed distinct, and that they can be distinguished by examples yet to be found, and which may well be more complicated than those given in this paper.

We have strengthened Bednarczyk's result that, in the absence of auto-concurrency, RI-IB is as strong as H-H bisimulation (Bednarczyk 1991), by showing that we need only exclude events with equidepth auto-concurrency (Theorem 9.7). In Figure 22 we have given the full hierarchy of forward and reverse bisimulations for configuration structures with no equidepth auto-concurrency.

Bednarczyk also asked whether RS-SB was as strong as H-H. We can now answer his question in the negative as can be seen in Example 4.7. We moreover observe that there is a proper hierarchy in Figure 24 of reverse bisimulations that places RP-PB between $\mathrm{H}-\mathrm{H}$ and RS-SB, namely $\approx_{h-h} \subsetneq \approx_{r p-p b} \subsetneq \approx_{r s-s b} \subsetneq \approx_{r i-i b}$ (see also Figure 25). The strongest reverse equivalence short of full $\mathrm{H}-\mathrm{H}$ is $\mathrm{H}-\mathrm{WHPB}$, which has full reverse power. Example 4.8 shows that it is strictly less powerful than $\mathrm{H}-\mathrm{H}$. Thus in a sense we need a "forward" component as well as a "reverse" one.

We have shown in Proposition 5.25 that all the power of RS-SB equivalence resides in the reverse step transitions, with the forward steps being dispensable (can be replaced with single-event transitions), namely RS-SB and RS-IB coincide. In fact, the reverse steps can be restricted to those which are homogeneous (Theorem 5.10). One can even 


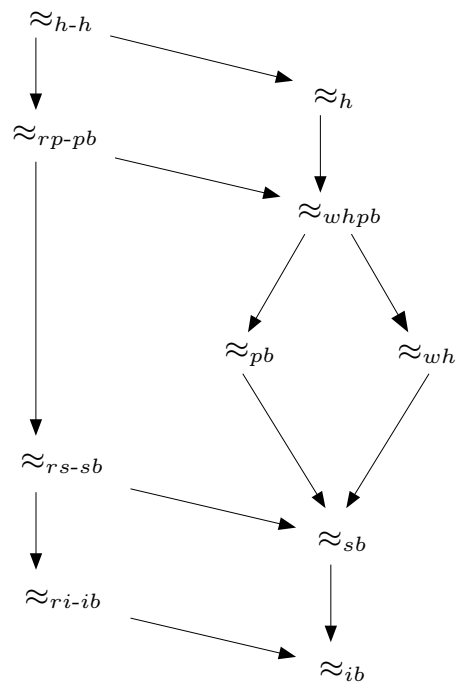

Fig. 25. The hierarchy of main bisimulations.

restrict attention to reverse homogeneous equidepth steps, where all events have the same depth (Theorem 5.24).

We have also proved that RS-SB equivalence preserves depth, in the sense that corresponding events must have the same depth. We have introduced the notion of depthrespecting bisimulation (DB). In the forward-only direction, it turns out that DB is strictly stronger than SB. However we have proved that reverse depth-respecting DB (RD-DB) has the same power as RS-SB (Theorem 5.24).

We have also investigated which of our reverse-type equivalences are preserved by refinement of actions. van Glabbeek and Goltz showed that history-preserving bisimulation and hereditary history-preserving bisimulation are preserved by refinement. We have rounded out their study by showing (Proposition 8.1) that all the reverse-type equivalences considered here fail to be preserved by refinement, with the possible exception of those few ones which are sandwiched between $\mathrm{H}$ and $\mathrm{H}-\mathrm{H}$.

The full hierarchy of equivalences can be distilled into a hierarchy of important equivalences presented in Figure 25. All main forward-only equivalences studied in the literature are present as well as the four major reverse bisimulations studied here. Three of the reverse bisimulations are based on observations: single-event transitions (RI-IB), step transitions (RS-SB), and pomset transitions (RP-PB); by contrast $\mathrm{H}-\mathrm{H}$ is based on the hereditary history-preserving property, which we do not consider as observable. All inclusions presented in Figure 25 are proper and there are no other inclusions perhaps with one exception: it is not known in general if an RS-SB is a WH. If one is interested in reverse bisimulations defined in terms of observations, then all equivalences in Figure 25 are suitable candidates except $\mathrm{H}$ and $\mathrm{H}-\mathrm{H}$. In contrast, if an equivalence is sought that is preserved by action refinement, then only $\mathrm{H}$ and $\mathrm{H}-\mathrm{H}$ are useful (Proposition 8.1).

In Figure 26 we list all examples used in the paper, together with which equivalences 


\begin{tabular}{lll}
\hline Example & Valid & Invalid \\
\hline Example 3.12 & ib, ri-ib & hsb, (sb), hesb \\
Example 3.13 & sb, ri-sb, ri-esb & pb, db, rs-ib \\
Example 3.14 & pb & db, ri-ib \\
Example 3.15 & wh & pb, ri-ib \\
Example 3.16 & whpb & h, ri-ib \\
Example 3.22 & db & pb, wh, ri-ib \\
Example 3.26 & hsb, hesb & sb, esb, ri-ib \\
Example 3.27 & esb & hsb, ri-ib \\
Example 3.29 & sb, ri-ib & hesb, pb, ri-sb \\
Example 3.30 $(\mathcal{E}, \mathcal{F})$ & pb, esb & db, ri-ib \\
Example 3.30 $\left(\mathcal{E}^{\prime}, \mathcal{F}^{\prime}\right)$ & pb, ri-pb & hesb \\
Example 4.5 & ri-pb, h & ri-db \\
Example 4.6 & ri-pb & db \\
Example 4.7 & rp-sb, h-wh & pb \\
Example 4.8 & $\mathrm{~h}$-whpb & $\mathrm{h}$ \\
Example 4.9 & $\mathrm{~h}$ & ri-ib \\
Figure 17 & ri-hesb & esb, (db), (ri-esb) \\
Example 9.8 & pb & esb \\
Example 9.9 & sb, hesb & pb, esb, ri-ib \\
\hline
\end{tabular}

Fig. 26. Examples

are valid or invalid. We list the relevant strongest equivalences in the Valid column and the weakest equivalences in the Invalid column with a convention that parentheses mean that the equivalence is implied by another equivalence. To save clutter we write, e.g. "db" instead of $\approx_{d b}$.

\section{Acknowledgements}

The second author acknowledges partial support by EPSRC grant EP/G039550/1 and JSPS grants S-09053 and FU-019. He also thanks the University of Leicester for granting study leave. We are grateful to the MSCS and SOS 2009 referees for their helpful comments and suggestions. We would also like to thank Pedro d'Argenio for suggesting looking at the additional combinations of forward and reverse observations to complete the hierarchy.

\section{References}

S. Abramsky. Observation equivalence as a testing equivalence. Theoretical Computer Science, 53:225-241, 1987.

M.A. Bednarczyk. Hereditary history preserving bisimulations or what is the power of the future perfect in program logics. Technical report, Institute of Computer Science, Polish Academy of Sciences, Gdańsk, 1991.

G. Boudol and I. Castellani. On the semantics of concurrency: partial orders and transition 
systems. In Proceedings of TAPSOFT'87, volume 249 of Lecture Notes in Computer Science, pages 123-137. Springer-Verlag, 1987.

F. Cherief. Back and forth bisimulations on prime event structures. In Proceedings of PARLE '92, volume 605 of Lecture Notes in Computer Science, pages 843-858. Springer-Verlag, 1992.

R. De Nicola, U. Montanari, and F. Vaandrager. Back and forth bisimulations. In Proceedings of CONCUR '90, Theories of Concurrency: Unification and Extension, volume 458 of Lecture Notes in Computer Science, pages 152-165. Springer-Verlag, 1990.

R. De Nicola and F. Vaandrager. Three logics for branching bisimulation (extended abstract). In Proceedings, Fifth Annual IEEE Symposium on Logic in Computer Science, pages 118-129. IEEE, Computer Society Press, 1990.

P. Degano, R. De Nicola, and U. Montanari. Observational equivalences for concurrency models. In M. Wirsing, editor, Formal Descriptions of Programming Concepts - III, Proceedings of the 3rd IFIP WG 2.2 Conference, pages 105-129. North-Holland, 1987.

H. Fecher. A completed hierarchy of true concurrent equivalences. Information Processing Letters, 89(5):261-265, 2004.

S.B. Fröschle. Decidability and Coincidence of Equivalences for Concurrency. PhD thesis, University of Edinburgh, 2004.

S.B. Fröschle and S. Lasota. Decomposition and complexity of hereditary history preserving bisimulation on bpp. In Proceedings of CONCUR 2005, volume 3653 of Lecture Notes in Computer Science, pages 263-277. Springer-Verlag, 2005.

R.J. van Glabbeek. History preserving process graphs. Draft 20 June 1996. http://boole. stanford.edu/ rvg/pub/history.draft.dvi, 1996.

R.J. van Glabbeek and U. Goltz. Partial order semantics for refinement of actions - neither necessary nor always sufficient but appropriate when used with care. Bulletin of the EATCS, 38:154-163, 1989.

R.J. van Glabbeek and U. Goltz. Refinement of actions and equivalence notions for concurrent systems. Acta Informatica, 37(4/5):229-327, 2001.

R.J. van Glabbeek and F.W. Vaandrager. The difference between splitting in $n$ and $n+1$. Information and Computation, 136(2):109-142, 1997.

A. Joyal, M. Nielsen, and G. Winskel. Bisimulation from open maps. Information and Computation, 127(2):164-185, 1996.

M. Jurdziński, M. Nielsen, and J. Srba. Undecidability of domino games and hhp-bisimilarity. Information and Computation, 184(2):343-368, 2003.

R. Milner. Communication and Concurrency. Prentice-Hall, 1989.

M. Nielsen and C. Clausen. Bisimulation, games, and logic. In Results and Trends in Theoretical Computer Science, volume 812 of Lecture Notes in Computer Science, pages 289-306. Springer-Verlag, 1994.

M. Nielsen, G.D. Plotkin, and G. Winskel. Petri nets, event structures and domains, part I. Theoretical Computer Science, 13:85-108, 1981.

I.C.C. Phillips and I. Ulidowski. Reversibility and models for concurrency. In M.C.B. Hennessy and R. van Glabbeek, editors, Proceedings of Fourth International Workshop on Structural Operational Semantics (SOS 2007), volume 192(1) of Electronic Notes in Theoretical Computer Science, pages 93-108. Elsevier, Amsterdam, 2007.

I.C.C. Phillips and I. Ulidowski. Reversing algebraic process calculi. Journal of Logic and Algebraic Programming, 73(1-2):70-96, 2007.

I.C.C. Phillips and I. Ulidowski. Reverse bisimulations on stable configuration structures. In B. Klin and P. Sobociński, editors, Proceedings of Sixth International Workshop on Structural Operational Semantics (SOS 2009), volume 18 of Electronic Proceedings in Theoretical Computer Science, pages 62-76. EPTCS, 2010. 
L. Pomello. Some equivalence notions for concurrent systems - An overview. In Advances in Petri Nets 1985, volume 222 of Lecture Notes in Computer Science, pages 381-400. SpringerVerlag, 1986.

A. Rabinovich and B.A. Trakhtenbrot. Behavior structures and nets. Fundamenta Informaticae, 11(4):357-403, 1988.

G. Winskel. Event structures. In Advances in Petri Nets 1986, volume 255 of Lecture Notes in Computer Science, pages 325-392. Springer-Verlag, 1987. 